\title{
Optical vortex induction via light-matter interaction in liquid-crystal media
}

\author{
R. Barboza, ${ }^{1}$ U. Bortolozzo, ${ }^{2}$ M. G. Clerc, ${ }^{1, *}$ S. Residori, ${ }^{2}$ and \\ E. Vidal-Henriquez ${ }^{1}$
}

${ }^{1}$ Departamento de Física, FCFM, Universidad de Chile, Casilla 487-3, Santiago, Chile

${ }^{2}$ INLN, Université de Nice-Sophia Antipolis, CNRS, 1361 Route des Lucioles, 06560 Valbonne, France

*Corresponding author: marcel@dfi.uchile.cl

Received March 27, 2015; revised August 2, 2015; accepted August 3, 2015; published September 14, 2015 (Doc. ID 237082)

Optical vortices are attracting much attention because of their applications in various fields of optical processing, communications, enhanced imaging systems, and bio-inspired devices. Different methods to generate optical vortices have been devised to date. Among them, several are based on the exploitation of light-matter interaction in liquid-crystal media. This review focuses on the main mechanisms of vortex generation in liquid crystals, in particular on vortex generation induced by umbilics, that is, defects arising in homeotropically aligned nematic cells, which act as topological matter templates able to realize the conversion from spin to orbital angular momentum of an incoming Gaussian beam. Optical vortex induction in a photosensitive light valve is presented as a self-stabilizing mechanism leading to reconfigurable and programmable vortex arrays. On each lattice site, every matter vortex acts as a photonic spin-to-orbital momentum coupler, converting input arrays of circularly polarized beams into output arrays of vortex beams with topological charges consistent with the matter lattice symmetry. (C) 2015 Optical Society of America

OCIS codes: (050.4865) Optical vortices; (160.3710) Liquid crystals; (160.5335) Photosensitive materials; (190.4360) Nonlinear optics, devices

http://dx.doi.org/10.1364/AOP.7.000635

1. Introduction. . . . . . . . . . . . . . . . . . . . . 637

1.1. Historical Motivations and Properties

of Optical Vortices. . . . . . . . . . . . . . . . 637

1.2. Generation of Optical Vortices . . . . . . . . . . . 638

1.3. Aim and Outline . . . . . . . . . . . . . . . . . 639

2. Optical Vortex Generation by Inhomogeneously Aligned Nematic Liquid

Crystals . . . . . . . . . . . . . . . . . . . . . . . 640

2.1. Inhomogeneously Aligned Nematic Liquid Crystal Cells: q-Plates . . 640

2.2. Nematic Liquid Crystal Droplets . . . . . . . . . . . . . . 642 
2.3. Umbilics in Homeotropically Aligned Nematic

Liquid Crystal Cells. . . . . . . . . . . . . . . . . . . . . . 643

3. Umbilics as Dissipative Vortices . . . . . . . . . . . . . . . . . . 645

3.1. Vortex Gas: Coarsening Dynamics . . . . . . . . . . . . . 646

4. Vortex Induction Mediated by Light-Matter Interaction . . . . . . . . 647

4.1. Homeotropic LCLV . . . . . . . . . . . . . . . . . . . . 648

4.2. Selective Vortex Induction. . . . . . . . . . . . . . . . . . 649

5. Vortex Induction Provides Photo-Addressable

q-Plates . . . . . . . . . . . . . . . . . . . . . 650

5.1. Induction of the Opposite Topological Charge . . . . . . . . . 651

6. Origin of the Vortex Induction Via Anisotropy Stabilized Light-Matter

Interaction. . . . . . . . . . . . . . . . . . . . . 652

6.1. Amplitude Equation Close to the Fréedericksz Transition for an

LCLV . . . . . . . . . . . . . . . . . . . 653

6.2. Positive Vortex with Swirling Arms . . . . . . . . . . 656

7. Vortex Arrays in Various Liquid-Crystal Systems . . . . . . . . . . 659

7.1. Torons in Photosensitive Frustrated Chiral Nematic

Liquid Crystals . . . . . . . . . . . . . . . . . . . . 659

7.2. Self-Assembled Defects in Smectic Liquid Crystals. . . . . . . . 661

7.3. Umbilics in Nematics Driven by Microarrays of Electrodes . . . 662

7.4. Umbilics in Nematics Driven by Magnetic Fields . . . . . . . . . 662

8. Programmable Optical Vortex Lattices in a Photo-Addressable

Homeotropic LCLV . . . . . . . . . . . . . . . . . . . . . . . . 664

8.1. Addressing Different Vortex Lattice Symmetries . . . . . . . . . . 664

8.2. Random Lattices: Dynamics of the Vortex Realignment. . . . . . . 667

9. Conclusions and Outlook . . . . . . . . . . . . . . . . . 667

Appendix A: Derivation of the Model Description . . . . . . . . . . 668

A.1. Derivation of the Amplitude Equation Close to the Fréedericksz

Transition . . . . . . . . . . . . . . . . . . 668

A.2. Isotropic Limit, the Ginzburg-Landau Equation with Real

Coefficients . . . . . . . . . . . . . . . . . . . 670

A.3. Anisotropic Ginzburg-Landau Equation with Real Coefficients: on

the Nature of the Positive and Negative Vortices . . . . . . . . 672

Acknowledgment. . . . . . . . . . . . . . . . . . . . . . . 677

References . . . . . . . . . . . . . . . . . . . . 678 


\title{
Optical vortex induction via light-matter interaction in liquid-crystal media
}

\author{
R. Barboza, U. Bortolozzo, M. G. Clerc, S. Residori, and \\ E. Vidal-Henriquez
}

\section{Introduction}

\subsection{Historical Motivations and Properties of Optical Vortices}

Inspired by the unexpected intricate structures of radio echoes from the bottom of the Antarctic ice sheet [1-3], Nye and Berry conducted ultrasound pulse experiments on a rough surface, allowing them to establish the emergence of singularities in wave trains [4], which correspond to dislocations in the wave train. In optics this wave train dislocation is detected by means of Mach-Zehnder interferometry [5]. Qualitatively, these wave train dislocations correspond to the junction of two regions with different numbers of stripes. The concept of dislocation is taken from the framework of material science, where a dislocation is a crystallographic defect, or irregularity, within a crystal structure [6], which adds or removes a line of atoms (stripe) in the crystal lattice.

Likewise, if one considers the complex envelope of the wave train, dislocations correspond to point-like singularities, a vortex solution [7], which locally break the symmetry. They exhibit a zero intensity at the singular point with a phase spiraling around it. Hence, optical vortices are singular points where the electromagnetic field goes to zero and around which the phase forms an $n$-armed spiral profile, with $n$ the topological charge; see [7-10] and the references therein. The origin of the topological charge is that the wave train is described by a complex scalar envelope having its normalized value (when it is defined) spanning the whole unit circle. The vortex position corresponds to the intersection of the zerolevel lines of real and imaginary parts of the complex envelope amplitude. This is a point where the phase is not defined, and this is known as phase singularity; however, the circulation of the phase around this point is well defined by $2 \pi n$ with $n= \pm 1, \pm 2, \ldots$. This integer number accounts for the topological charge or winding number of the vortex. Two vortices connected through their zerolevel curves have opposite charges; namely, the growth direction of the phase is opposite in each of the connected vortices. Therefore, if the envelope is a smooth function in a plane of the wavefront, then the vortices can be created and destroyed only as pairs.

It is well known that light can exert mechanical effects on matter. This is not limited to the effect of linear momentum radiation, which can cause particle translation. Particles can be set in rotation if the radiation is circularly polarized. In this case, the electromagnetic wave couples its spin angular momentum with that of the medium under interaction, and this effect is physically observable by the spinning of the particles around themselves or the object around itself, with the sense and rate of rotation depending on the helicity of the light beam. The 
first experimental observation of the fact that light can possess an intrinsic angular momentum was reported by Beth [11], who devised an experiment in which a polarized beam is sent onto a birefringent plate free to rotate. The plate is a half-wave plate at the operating wavelength and transforms a left-circularpolarized light beam in a right-circular-polarized beam. The plate experiences a torque that changes its sign with the input polarization. By measuring the amount of torque, the value of the angular momentum of the photon can be calculated if the incoming light intensity is known. The result was $\hbar$ for lefthand-polarized input and $-\hbar$ for the right-hand one. For intermediate values it was shown that torque diminishes and vanishes for linearly polarized beams. In addition to these effects, it was also observed that linearly polarized beams can, like Laguerre-Gauss beams, set particles in rotation and that they rotate around the axis of the beam and not around their own axis, as for a circularpolarized beam $[12,13]$. This is related to the so-called orbital angular momentum of the beam and its connection to the beam structure, the phase (see review article [14] and references therein).

Optical vortices have also been introduced on symmetry grounds as the topological defects arising above the laser transition [15]. In this context they have been identified as topological defects, that is, point-like phase singularities that locally break the symmetry of a rotationally invariant complex field and that appear and disappear in pairs of opposite charge. They have been reported in several experiments, as in photorefractive cavities [16] and in lasers [17,18].

In Laguerre-Gauss beams, a single optical vortex corresponds to a phase singularity on the axis of the beam [19]. It has been shown that Laguerre-Gauss modes carry an orbital angular momentum $l \hbar$ per photon, where $l$ is the topological charge of the phase singularity [20]. Optical vortex beams attract a lot of attention in view of their applications, including the exchange of angular momentum between light and matter [20], optical tweezers [21-23], high precision micromachining [24-26], quantum computation [27], data transmission [28-31], and the enhancement of astronomical images [32,33]. The ability to generate and manipulate vortex beams is, therefore, one of the key topics in current photonics. A recent review of the origins and applications of optical vortices can be found in [19] and references therein.

\subsection{Generation of Optical Vortices}

Different methods to generate optical vortices have been proposed: spiral phase plates [34], diffractive elements [35], computer-generated holograms [36], segmented deformable mirrors [37], and nanostructured glass plates [38].

Optical vortex generation by liquid crystals was initially proposed in the context of cholesteric liquid crystals, where dislocations in the stripe pattern of the cholesteric texture were used to produce diffraction orders with topological charges [39]. Recently, the introduction of q-plates, planar elements with a preset azimuthal orientation in nematic liquid crystals [40], has opened promising new avenues in the field of optical vortex generation by means of liquid-crystal media. Other recent experiments deal with the exploitation of the radial director distribution in liquid-crystal droplets [41] or umbilic-like defects in nematic textures $[42,43]$. All these approaches provide tunability and high efficiency to the vortex generation process, although liquid-crystal misalignment can cause some beam deformation and a consequent degradation of the generated optical vortices [44]. 
Recently, by exploiting re-orientational nonlinearities in the nematic liquidcrystal layer of an optically addressable liquid-crystal cell, a so-called light valve, the optically addressed self-induction of vortex-beams that are, by nature, self-aligned with the impinging light beam has been demonstrated [45]. Indeed, the spontaneous character of the induction process guarantees that the generated defect is aligned with the incoming light beam. In this framework, optical vortices derive directly from the induced umbilical defects in the liquid-crystal texture. The umbilic is a defect that naturally possesses a vortex-like morphology, making it attractive for spontaneously enabling the matter template to impress a helical structure on an incoming light wavefront. Based on the vortex induction process, the realization of programmable lattices of optical vortices with arbitrary distributions in space has been demonstrated [46]. Vortex lattices can also be obtained with other liquid-crystal systems, as confined cholesterics [47,48], smectic cells [49-51], nematics driven by segmented electrodes [52], and nematics driven by magnetic fields [53].

\subsection{Aim and Outline}

The aim of this paper is to review the main mechanisms for vortex generation in liquid-crystal media. Particular emphasis is made for nematic liquid crystals and umbilics, the defects arising in homeotropically aligned cells, which act as topological matter templates that can realize the conversion from spin to orbital angular momentum of an incoming Gaussian beam. Optical vortex induction is extensively presented as a self-stabilizing mechanism occurring in optically addressed liquid-crystal light valves (LCLVs). The properties of the induced optical vortices are characterized in this type of system, where the emergence of phase singularities is accomplished by sending circularly polarized light beams onto a homeotropic nematic liquid-crystal cell with a photosensitive wall.

Thanks to the photosensitive wall, local illumination impinging onto the light valve can induce a spontaneous matter vortex that remains stable and trapped at the chosen location. In turn, the coupling between the beam and the matter vortex generates an optical vortex with a topological charge consistent with angular momentum conservation. Theoretically, based on bifurcation theory, and close to the Fréedericksz transition [54-56], a forced Ginzburg-Landau equation can be used to describe the system. The mechanism for matter vortex generation and stabilization is provided by the concurrency of light-induced voltage gradients and anisotropy of the elasticity that characterizes the deformation of the liquidcrystal medium. Based on the self-stabilizing mechanism, programmable lattices of optical vortices are realized with arbitrary distributions in space. On each lattice site, every matter vortex acts as a photonic spin-to-orbital momentum coupler, so that input arrays of circularly polarized beams are converted into output arrays of vortex beams with topological charges consistent with the matter lattice.

The paper is organized as follows. Section 2 presents the main mechanisms of optical vortex generation in nematic liquid crystals, namely, q-plates, nematic droplets, and defects generated in homeotropically aligned nematic liquid-crystal cells. In Section 3, we discuss the dissipative character of vortex defects obtained by this latter method, due to the coarsening dynamics undergone by the vortex gas after its origin at the Fréedericksz transition. Section 4 is dedicated to vortex induction mediated by light-matter interaction in LCLVs. Section 5 
presents the vortex induction process as a method to achieve photo-addressable q-plates. In Section 6 the origin of vortex induction via anisotropy stabilized light-matter interaction is derived by model equations that describe the positive vortex and its swirling arm dynamics. Section 7 presents various methods for generating vortex arrays in different liquid-crystal systems, while Section 8 focuses on the programmable optical vortex lattices in a photo-addressable homeotropic LCLV. Finally, in Section 9 are presented the conclusions. Appendix A contains the detailed derivation of the model description.

\section{Optical Vortex Generation by Inhomogeneously Aligned Nematic Liquid Crystals}

Liquid crystals are a state of matter that shares the structures and properties of liquid and crystalline states (see, for instance, [54,55] and references therein). The main features of liquid crystals are that they have the ability to flow like liquids but also display symmetries inherited from crystalline solids. In given ranges of temperature, liquid crystals form mesophases, that is, intermediate phases between solids and liquids characterized by a certain level of order and characteristic organizations of the molecular arrangements. Among them, the most known are the nematic, cholesteric, smectic, and blue phases [54-56]. Liquid crystals are very attractive from the viewpoint of optics because of their high birefringence, optical transparency over a wide range of optical wavelengths, high sensibility to externally applied electric/magnetic fields, and their ability to provide strong coupling between matter and electromagnetic fields, generating changes in the polarization of light and matter [57,58].

Nematics are the most widely used liquid crystals for practical applications, such as for light modulators and liquid-crystal displays. Nematic liquid crystals are uniaxial mesophases that present a local orientational order defined by a unit vector $\vec{n}$, known as the nematic director. Therefore, they behave optically as uniaxial birefringent media. The director orientation can be controlled externally through electrical bias, optical fields, or surface interactions. We will see in the following sections that radial or twisted arrangements of the nematic director can be induced by different methods, leading to the corresponding generation of wave plates with varying optical axes.

\subsection{Inhomogeneously Aligned Nematic Liquid Crystal Cells: q-Plates}

A q-plate is a specifically designed nematic liquid-crystal cell in which a radial or twisted alignment of the molecules is obtained by rubbing circularly the glass plates forming the cell [40]. The distribution of the nematic director follows the radial or twisted conditions imposed at the anchoring surfaces and, therefore, produces an inhomogeneous retardance wave plate with varying optical axis, as the optical axis follows the lines along which the molecules are aligned. The wave plate's ability to produce optical vortices is related to the winding number $q$ that characterizes the nematic director distribution, hence, the name "q-plate." Examples of q-plates with $q=1 / 2$ and $q=1$ are shown in Fig. 1.

The versatile manufacture and use of q-plates is particularly interesting because of their ability to perform spin-to-orbital angular momentum conversion, leading to the generation of helical modes. The topological charge of the generated 


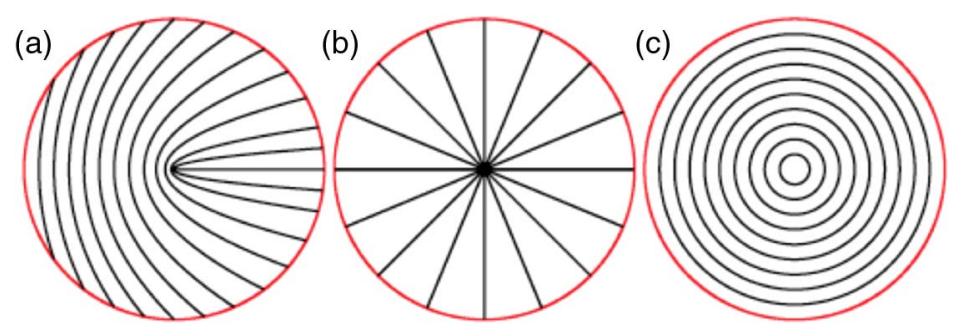

Examples of q-plates. The local direction of the optical axis is tangent to the lines. (a) $q=1 / 2$ and $\tilde{\theta}_{0}=0$, (b) $q=1$ with $\tilde{\theta}_{0}=0$, and (c) with $\tilde{\theta}_{0}=\pi / 2$. Reproduced with permission from Marrucci et al., Phys. Rev. Lett. 96, 163905 (2006) [40]. Copyright 2006 American Physical Society.

optical vortex is controlled by the $q$ number of the plate [59]. In fact, if we neglect diffraction inside the thin liquid-crystal layer (usually a few micrometers thick), and we consider only the transverse component of the electric field for the incoming optical beam, the action of the q-plate can be described by a Jones matrix, which takes the form

$$
M=\left[\begin{array}{ll}
1 & 0 \\
0 & 1
\end{array}\right] \cos \frac{\Delta}{2}+i\left[\begin{array}{cc}
\cos 2 \tilde{\theta} & \sin 2 \tilde{\theta} \\
\sin 2 \tilde{\theta} & -\cos 2 \tilde{\theta}
\end{array}\right] \sin \frac{\Delta}{2},
$$

with $\Delta$ the overall effective phase shift between the (local) ordinary and (local) extraordinary component of the electric field. $\Delta$ is given by $\Delta=2 \pi \frac{d}{\lambda}\left(\bar{n}-n_{o}\right)$, where $d$ is the thickness of the liquid-crystal layer,

$$
\bar{n}=\frac{1}{d} \int_{0}^{d} n(\beta) \mathrm{d} z
$$

and

$$
n(\beta)=\frac{n_{e} n_{o}}{\sqrt{n_{o}^{2} \sin ^{2} \beta+n_{e}^{2} \cos ^{2} \beta}}
$$

is the local refractive index seen by the extraordinary component of the electric field. Here, $\psi$ is the liquid-crystal tilt angle, $n_{o}$ and $n_{e}$ are, respectively, the ordinary and extraordinary refractive index of the liquid crystal, and $\beta, \tilde{\theta}$ are the polar and azimuthal coordinates of the nematic director.

We will assume for simplicity that $\tilde{\theta}=q \xi+\tilde{\theta}_{o}(r, z)$, where $\{r, \xi, z\}$ are the local cylindrical coordinates, and $\beta=\beta(r, z)$. For a circularly polarized input $\vec{e}_{\sigma}=A \frac{1}{\sqrt{2}}(\vec{x}+i \sigma \vec{y})$, where $\sigma=+1$ stands for left-handed (LH) and $\sigma=-1$ for right-handed $(\mathrm{RH})$ circular polarization, and dropping down irrelevant phase factors, it can be shown that the output beam is given by

$$
\vec{E}=A \cos \frac{\Delta}{2} \vec{e}_{\sigma}+\exp (2 i q \sigma \xi) A \exp \left(2 i \sigma \tilde{\theta}_{0}\right) \sin \frac{\Delta}{2} \vec{e}_{-\sigma}
$$

As it appears from Eq. (4), the incoming circularly polarized field is converted to a beam with opposite polarization and with an additional helical phase factor $\exp 2 i q \xi$ through a power conversion factor $\sin ^{2}(\Delta / 2)$. For example, a LH 
(RH) circularly polarized Gaussian input beam entering a q-plate with $q=1$ will exit, for the ideal case $\Delta=\pi$ (half-wave plate with nonuniform optical axis), with $\mathrm{RH}(\mathrm{LH})$ circular polarization and with an additional helical phase with winding number $m=+2(m=-2)$. The acquired helical phase profile changes the structure of the amplitude of the output field. Indeed, the amplitude has to go to zero at the center of the beam to have a single valued field, giving birth to an optical vortex beam.

A rapid check of the amount of the momentum transferred to the q-plate shows that we are in the presence of conversion of the spin angular momentum (from the input beam) to orbital angular momentum (to the output beam). Indeed, the angular momentum per photon for the input beam is $\sigma \hbar$, whereas for the output beam we have to count $\sigma \hbar \cos ^{2}(\Delta / 2)$ for the $\sigma$ polarized component (the spin part) and $-\sigma \hbar \sin ^{2}(\Delta / 2)+2 \sigma \hbar \sin ^{2}(\Delta / 2)$ for the $-\sigma$ polarized component (spin and orbital part). It appears clearly that the net change in the angular momentum is 0; hence, we are in the case of spin-to-orbital angular momentum conversion. Since the total torque density on the q-plate is proportional to the net change in the optical angular momentum [59], the incident beam does not exert any torque on the plate.

\subsection{Nematic Liquid Crystal Droplets}

Spherical droplets of a nematic liquid crystal have been created by dispersing the nematic liquid crystal 5CB in water [41]. The droplets are stabilized by a surfactant and have a controlled diameter of a few micrometers. Inside the droplet, the director, and hence the local optical axis, follows a spherical distribution. This is shown in Fig. 2, together with the mechanism of conversion of spin to orbital angular momentum for an incoming focused Gaussian beam. The

\section{Figure 2}

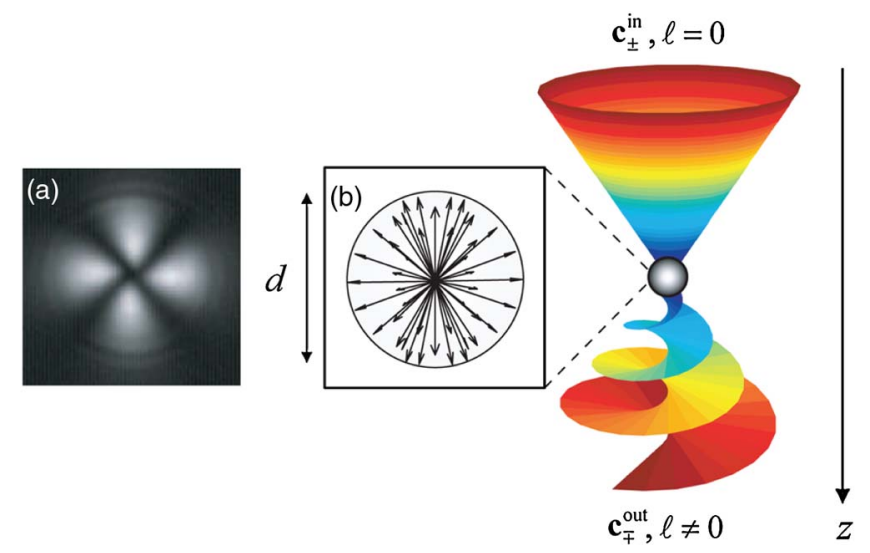

Optical vortex generation from a nematic liquid-crystal droplet. An incident light beam with circular polarization $c_{ \pm}^{\text {in }}$ and a smooth profile $(l=0)$ is partly converted into the orthogonal polarization state $c_{\mp}^{\text {out }}$ carrying a phase singularity $(l \neq 0)$. Inset $\mathrm{a}$, crossed polarizer image, and inset $\mathrm{b}$, radial structure of the liquid-crystal director showing the radial distribution inside the droplet. Reprinted with permission from Brasselet et al., Phys. Rev. Lett. 103, 103903 (2009) [41]. Copyright 2009 American Physical Society. 
spatial conformation of the optical axis inside the droplet defines an inhomogeneous birefringent medium with a defect at the center, as it appears from the pattern observed under cross polarizers [Fig. 2(a)]. When looking at the median plane, the texture is similar to that of a q-plate with $q=1$ and the director orientation around the defect that winds by $2 \pi$ [Fig. 2(b)]. When these droplets are optically trapped in tightly focused circularly polarized Gaussian beams, their natural on-axis positioning in the focal region leads the trapping beam to read out the radial birefringence distribution and to generate a spiraling wavefront (Fig. 2).

The trapping beam itself can, thus, be used for vortex generation: the beam traps the droplets on-axis and preserves their radial symmetry. Droplets with various diameters can be trapped using beams with various intensities. The efficiency of conversion of the input beam into the orthogonally polarized helical wavefront beam is almost independent of the optical power but depends on the size of the droplets. An increasing amount of the tightly focused incident Gaussian beam is converted into a vortex beam as the droplet size increases, typically, from 1.5 to $2.4 \mu \mathrm{m}$ [41]. The increase in efficiency can be related to the spatial overlap of the droplet and the Gaussian beam. At $3.2 \mu \mathrm{m}$, the subsequent decrease in the conversion efficiency can be attributed to the decrease of the averaged optical birefringence seen by the propagating beam [41].

\subsection{Umbilics in Homeotropically Aligned Nematic Liquid Crystal Cells}

Besides q-plates, one of the adopted methods for generating optical vortices with nematics relies on the exploitation of a fixed single topological defect, that is, a 3D orientational structure called "umbilical defect" or, in short "umbilic" [60]. Umbilics have long been reported in the literature [54-56] and have accompanied liquid crystals since their discovery in 1889 by Lehmann [61], who called these structures kernels. Later, they were observed in a similar experimental setup by Freidel, who also resolved their detailed topological structure and called them noyaux [62]. From the theory of elasticity, Frank calculated the detailed structure of these defects [63] and, because they break the orientational order, he called them disclinations by analogy with dislocations in crystals of condensed matter. However, the most widely used name to refer to these defects is nematic umbilical defects. The term umbilics was coined by Rapini [60] and refers to the topological structure of the defect corresponding to a string-like object in three dimensions. This is schematically represented in Fig. 3.

As described above, the nematic liquid-crystal phase is characterized by rodshaped molecules that have no positional order but tend to point in the same direction, described by a unit vector, the so-called nematic director $\vec{n}[54,55]$. In homeotropically aligned liquid-crystal cells, the director $\vec{n}$ is perpendicular to the confining walls. When a nematic liquid-crystal cell is biased and the voltage across the nematic layer is above the Fréedericksz transition voltage, the molecules reorient. This is because the torque stemming from the elastic forces linking together the liquid-crystal molecules is overcome by the electric torque due to the applied voltage. If the nematic liquid-crystal layer is homeotropically aligned and the dielectric anisotropy is negative, then the molecules will rotate perpendicularly to the applied electric field. Because of the $2 \pi$ degeneracy of the direction of the reorientation, the molecules will align in random directions, 
(a)

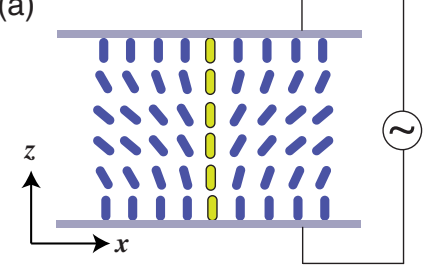

(b)

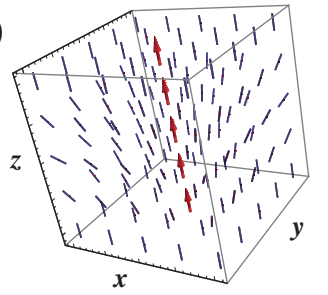

(a) Representation of the umbilical defect in an homeotropically aligned nematic liquid-crystal cell: rods represent the orientation of the director, the green (lighter gray) rods correspond to the position of the vortex core. (b) Three-dimensional view of the umbilic, where arrows stand for the position of the vortex core. Adapted from Fig. 1 in [64].

causing the formation of defects in the texture of the nematic layer. These defects are in general umbilics with winding number, or Frank index, \pm 1 [54].

The electro-optical properties of umbilics for generating optical vortices were initially demonstrated in $[42,43]$. Figure 4 displays the calculated profile for $\mathrm{a}+1$ umbilic defect used for the purpose of optical vortex generation [43]. From the 2D section of the director field we can note that the molecular arrangement around the defect core is equivalent to a q-plate with $q=1$. Hence, the umbilic creates automatically the required wave plate for the generation of an optical vortex with topological charge $m=2$.

\section{Figure 4}
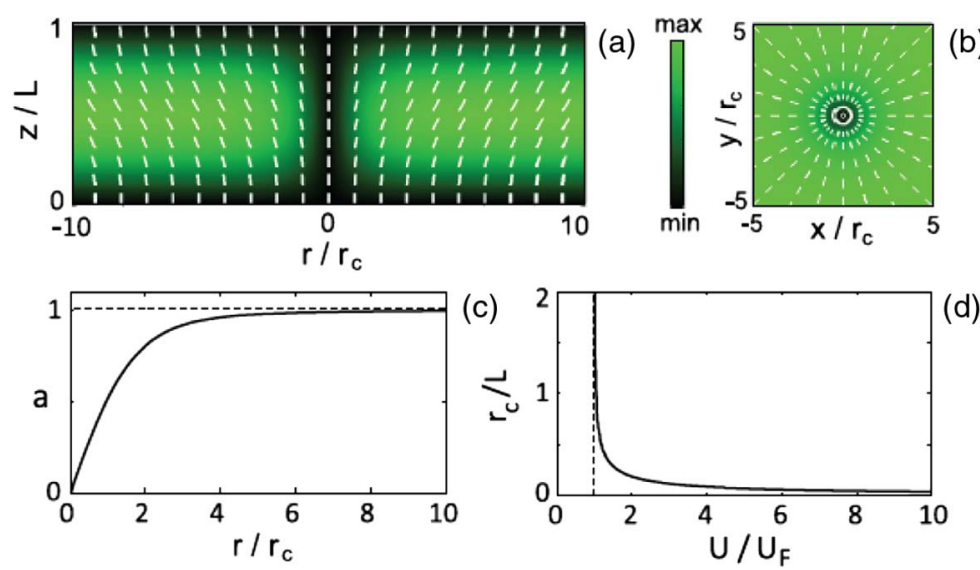

(a) Longitudinal and (b) transverse cross sections of the director field of $a+1$ umbilic defect; $r_{c}$ is the core radius of the defect and $L$ is the nematic film thickness. (c) Calculated reduced amplitude a of the tilt angle nearby the defect located at $r=0$. (d) Calculated reduced core radius $r_{c} / L$ versus reduced voltage $U / U_{F}$, where $U$ is the voltage applied to the liquid-crystal cell and $U_{F}$ is the Fréedericksz transition voltage. Reprinted with permission from Brasselet, Phys. Rev. Lett. 108, 087801 (2012) [43]. Copyright 2012 American Physical Society. 
Because of the complex elasticity theory associated with nematic liquid crystals, characterized by three types of deformation (bend, twist, and splay), the study of these defects from the dynamical point of view is a thorny task [54-56]. A simple and universal strategy is to analyze their behavior near the orientational instability of the molecules that occurs at the Fréedericksz transition [54,55]. Indeed, close to this transition the dynamics of the director can be reduced at main order to the Ginzburg-Landau equation with real coefficients $[65,66]$ (a detailed explanation of the derivation of this equation is presented in Appendix A):

$$
\partial_{t} A=\mu A-|A|^{2} A+\nabla_{\perp}^{2} A,
$$

where $\mu$ is the bifurcation parameter describing the distance from the Fréedericksz transition voltage, $\nabla_{\perp}$ is the transverse Laplacian, and $A$ is the complex amplitude describing the projection of the director field on the $x-y$ plane.

This amplitude equation allows an understanding of the emergence of different orientational domains, two types of stable vortices with $\pm 2 \pi$ phase jump, corresponding to winding number \pm 1 , and their respective dynamics. In this approach, however, both defects are indistinguishable in their amplitude and, as a result of the phase invariance of the Ginzburg-Landau equation, they account for a continuous family of solutions, parametrized by the phase.

A generalization of the Ginzburg-Landau equation can be derived by taking into account the anisotropy of the liquid-crystal elastic constants [66] (see Appendix A):

$$
\partial_{t} A=\mu A-A|A|^{2}+\nabla^{2} A+\delta \partial_{\eta \eta} \bar{A}
$$

where $\partial_{\eta} \equiv \partial_{x}+i \partial_{y}, \nabla^{2}=\partial_{\eta \bar{\eta}}$, and $\delta=\left(K_{1}-K_{2}\right) /\left(K_{1}+K_{2}\right)$, with $K_{1}$ and $K_{2}$ the splay and twist elastic constants of the nematic liquid crystal. This equation is the anisotropic Ginzburg-Landau equation. Appendix A.3 shows that this form of the amplitude equation allows discrimination between the positive and negative vortices. Indeed, the elastic energy associated with these two molecular reorientations is different [54], which is the physical origin of the asymmetry between these defects.

\section{Umbilics as Dissipative Vortices}

Far from equilibrium, systems with injection and dissipation of energy, momenta, and matter exhibit instabilities that lead to spontaneous symmetry breaking and pattern formation [67]. Due to the inherent fluctuations of these macroscopic systems, different states may emerge in distinct regions of space; hence, spatial structures are usually characterized by domains, separated by interfaces, as grain boundaries, defects, or dislocations [68,69]. Among others, defects in rotationally invariant $2 \mathrm{D}$ systems have the structure of vorticesdissipative vortices - and attract much attention because of their universal character. Indeed, dissipative vortices are solutions of the complex Ginzburg-Landau equation (CGLE), which describes a variety of physical systems: fluids, superfluids, superconductors, liquid crystals, fluidized anisotropic granular matter, magnetic media, and optical dielectrics, to mention a few [7]. 


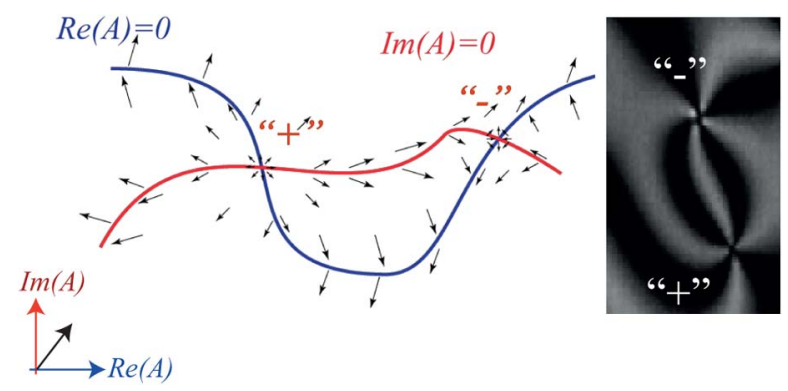

Topological description for the origin of a vortex. The vector field is represented by black arrows. The red (blue) curve corresponds to a field completely horizontal (vertical), i.e., represents a zero-level line $\operatorname{Im}(A)=0(\operatorname{Re}(A)=0)$. Interception of the zero-level lines leads to the generation of a vortex. The inset is an experimental image of a pair of umbilical defects observed under linear crossed polarizers. The symbols \pm account for their respective topological charge.

Nematic liquid crystals with negative anisotropic dielectric constant and homeotropic anchoring are a natural physical context where dissipative vortices are observed [54,55]. Two types of stable vortices with opposite charges are observed; these are characterized by being attracted (repulsed) to the opposite (identical) topological charge $[7,70,71]$.

The origin of the topological charge comes from the vectorial nature of the director field, which can be described by a complex scalar field $A$ with two components, the real, $\operatorname{Re}(A)$, and imaginary, $\operatorname{Im}(A)$, parts. As shown in Fig. 5, a phase singularity, or topological vortex, arises whenever the real and imaginary parts go simultaneously to zero. Then, the vortex position corresponds to the intersection of the real and imaginary zero-level curves. The phase $\tan \varphi=$ $\operatorname{Im}(A) / \operatorname{Re}(A)$ is not defined at this point, so we are in the presence of a phase singularity. The circulation of the phase around the zero-level lines' intersection is well defined and corresponds to an integer multiple of $2 \pi$ :

$$
\oint_{C} \nabla \varphi \cdot \mathrm{d} \vec{r}=2 \pi n
$$

with $n= \pm 1, \pm 2, \ldots$. This integer number accounts for the topological charge or winding number of the vortex.

\subsection{Vortex Gas: Coarsening Dynamics}

Two vortices connected through their zero-level curves have opposite charges, namely, the growth direction of the phase is opposite in each of the connected vortices, as represented in Fig. 5. Therefore, if the field is a smooth function the vortices can be created and destroyed only as pairs. Experimentally, the intensity of the light that emerges from a homeotropically aligned liquid-crystal sample between crossed polarizers exhibits similar properties to the field $\psi(\vec{r}, t)$ defined as $\psi \equiv \operatorname{Re}(A) \cdot \operatorname{Im}(A)$. We will call this scalar field a nullcline field. Figure 6(a) shows a vortex gas obtained by experimental snapshots recorded under white-light illumination for a homeotropic cell between crossed polarizers. 


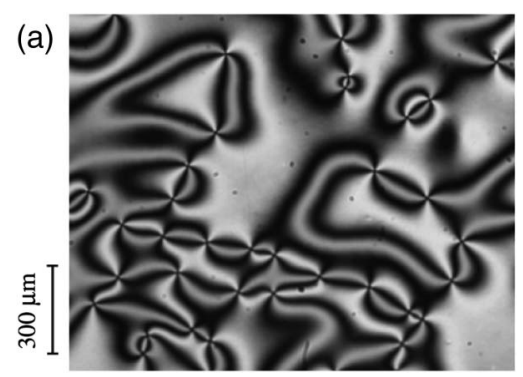

(b)

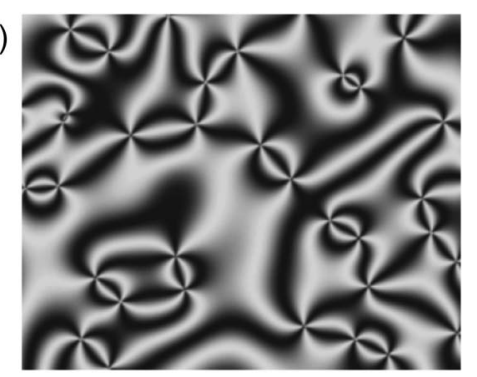

Vortex gas: (a) experimental snapshot recorded under white-light illumination for a homeotropically aligned liquid-crystal cell between crossed polarizers and for uniform laser illumination of the photosensitive wall, and (b) nullcline field $\psi$ obtained from numerical simulations of Eq. (5) for parameters corresponding to the experimental conditions. Reprinted from Barboza et al., New J. Phys. 15, 013028 (2013) [71]. C IOP Publishing. Reproduced with permission. All rights reserved.

For comparison, a picture obtained from numerical simulations of Eq. (5) is shown in Fig. 6(b).

We must note that, even though appealing for the creation of optical vortices, the position and the number of defects created by the Fréedericksz transition are uncontrollable, because the system is driven from the unstable condition through random fluctuations of the director around the homeotropic state. Umbilics with the same winding number will repel each other, and those with opposite winding number will attract and annihilate. This dissipative process takes place through a coarsening dynamics [7] during which the gas of vortices relaxes toward an equilibrium state that exhibits a uniform texture or a pair of defects (eventually, one defect of the pair can be hidden by boundary effects) [70]. The remaining umbilic can, then, be used to generate optical vortices [42,43]; however, the spontaneous relaxation dynamics of the vortex gas does not offer control of the system, limiting the ability to select the final vortex position.

\section{Vortex Induction Mediated by Light-Matter Interaction}

To overcome the difficulties and limitations related to the dissipative character of spontaneous defect generation in liquid-crystal samples, it is necessary to provide a controllable way to generate umbilics. The requirement is that the umbilics should be addressable by a specific control method, hence, by which they can be generated when needed and where required on the liquid-crystal cell.

For this purpose, we rely on the LCLV, whose behavior is detailed in the paragraph below. The cell is biased, initially, to have the effective voltage across the liquid-crystal layer slightly below the Fréedericksz transition voltage, with the intent to avoid the spontaneous formation of umbilics. When a light beam of Gaussian shape is directed onto the photoconductive side of the LCLV, the impedance of the photoconductive bismuth silicon oxide $\left(\mathrm{Bi}_{12} \mathrm{SiO}_{20}\right.$, BSO) decreases because of the photogenerated charges, making it more conductive where the intensity is larger. The effective voltage across the liquid-crystal layer 
acquires, therefore, a bell-shaped profile, higher in the center. By increasing the bias voltage or the beam intensity, the peak value of the effective voltage can, eventually, go beyond the Fréedericksz voltage. As a consequence, the molecules will tilt first toward the $x-y$ plane, but not randomly as in an uniformly biased cell (normal homeotropic one).

The radial symmetry of the voltage drop will give rise to a transverse component of the electric field, also with the same radial profile. This biases the system, making the nematic director follow the same symmetry while reorienting, and giving birth to a single singularity in the texture of the nematic layer $[45,46,72]$.

\subsection{Homeotropic LCLV}

The LCLV is schematically represented in Fig. 7(a). It is composed of a liquidcrystal layer between two slabs of $25 \mathrm{~mm} \times 25 \mathrm{~mm}$ transverse size. The input face is made of a transparent photoconductor BSO with $1 \mathrm{~mm}$ thickness, whereas the other face is made of a glass plate of the same surface and $0.7 \mathrm{~mm}$ thickness. The interior surface of the glass plate and the external surface of the BSO are coated with indium tin oxide (ITO), a commonly used transparent conductor. The uncoated face of the BSO and the coated face of the glass plate are treated to provide homeotropic anchoring conditions. The LCLV is, then, assembled with $15 \mu \mathrm{m}$ spacers and filled with a nematic liquid crystal (MLC6608) with negative dielectric anisotropy $\varepsilon_{a}=\varepsilon_{\|}-\varepsilon_{\perp}=-4$.2, with $\varepsilon_{\|}$and $\varepsilon_{\perp}$ the dielectric susceptibility for low-frequency electric fields parallel and orthogonal,

\section{Figure 7}
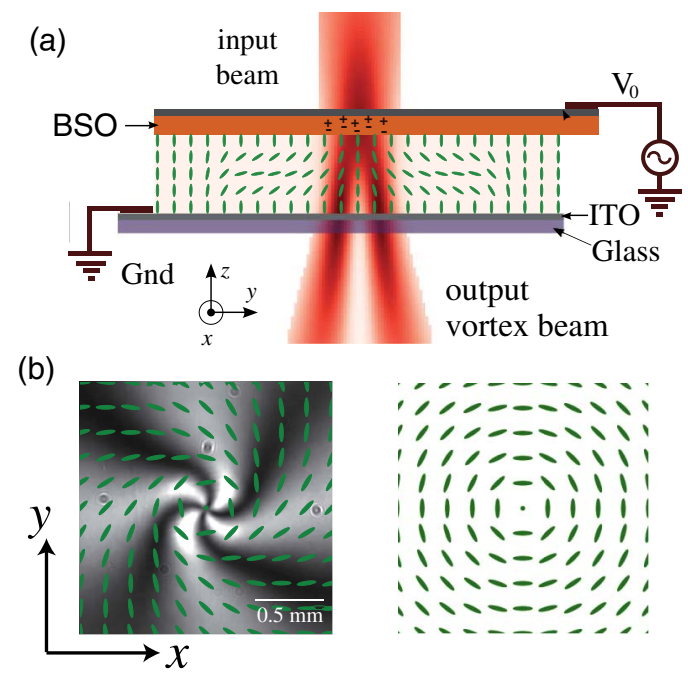

(a) Vortex induction in the homeotropic LCLV: a circularly polarized beam is incident on the photoconductive BSO side of the LCLV; the voltage $V_{0}$ is such that only the illuminated region undergoes the Fréedericksz transition. When reorienting, the liquid-crystal molecules follow the circular pattern associated with the electric field and create a matter vortex, which, in turn, induces a helical wavefront at the exit of the sample. (b) Umbilic-like defect exhibiting swirling arms with superposed molecular organization (left panel); for comparison, the molecular organization of a defect with straight cross arms is shown in the right panel. Adapted from Fig. 1 in [72]. 
(a)
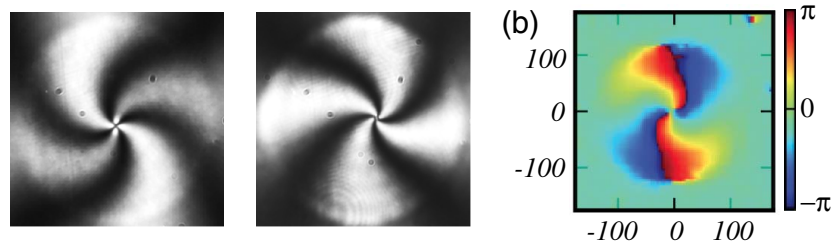

Matter vortices. (a) Intensity profiles recorded under white light illumination and crossed polarizers showing clockwise (left panel) and counterclockwise (right panel) swirling arms. (b) Spatially resolved polarimetry in the case of a defect with counterclockwise swirling arms. Adapted from Fig. 2 in [72].

respectively, to the molecular director. The LCLV is biased with a low-frequency (sine wave) voltage and the frequency is tuned to $100 \mathrm{~Hz}$ to obtain an optimal response to the incoming light. The ordinary and the extraordinary refractive indices are, respectively, $n_{o}=1.4637$ and $n_{e}=1.5338$, giving a maximum reachable phase shift of about $3.3 \pi$ at $633 \mathrm{~nm}$ and for a thickness $d=15 \mu \mathrm{m}$ of the liquid-crystal layer.

The photoconductive BSO layer acts as an optical tunable impedance producing a voltage divider arrangement that enables us to spatially tune the effective voltage across the liquid-crystal layer by using the proper illumination profile. As an example, in Fig. 7(b) is depicted the local distribution of the director under local illumination with a Gaussian beam over the experimental snapshot of a single umbilic defect obtained with this method. A characteristic feature of the observed vortex is that its arms are bent and, thus, they swirl around the defect core. For comparison, the molecular organization of a defect with straight cross arms is shown in the right panel of Fig. 7(b).

Experimental snapshots showing the matter defect with clockwise and counterclockwise swirling arms are displayed in the left and right panels, respectively, of Fig. 8(a). Spatially resolved polarimetry [73] allows discrimination of the sign of the defect. The local birefringence is analyzed by using quarter-wave plates, allowing the reconstruction of the director distribution around the defect, as shown in Fig. 8(b). The obtained polarimetric profile shows that the defect is, indeed, umbilic-like and of winding number +1 . Note that the reconstructed phase is $2 \theta$, with $\theta$ the liquid-crystal tilt in the transverse plane; therefore, the $4 \pi$ phase jump around the singularity indicates a $2 \pi$ change of the liquid-crystal tilt angle $\theta$. The swirling of the arms appears also from the polarimetric profile. This is a characteristic feature of the umbilic and can be explained on the basis of energetic arguments, as described in the following sections.

\subsection{Selective Vortex Induction}

The experimental setup for the selective induction of vortices in the LCLV is shown in Fig. 9(a). A circularly polarized Gaussian beam, either RH LH, with a waist of $395 \mu \mathrm{m}$ is sent onto the LCLV. The setup is arranged to have a MachZehnder interferometer with the LCLV on one arm for the visualization of the optical wavefront at the exit of the LCLV. A quarter-wave plate and a polarizer are placed after the LCLV to filter out the incoming circular beam, so that at $0 \mathrm{~V}$ the measured output intensity is 0 . 

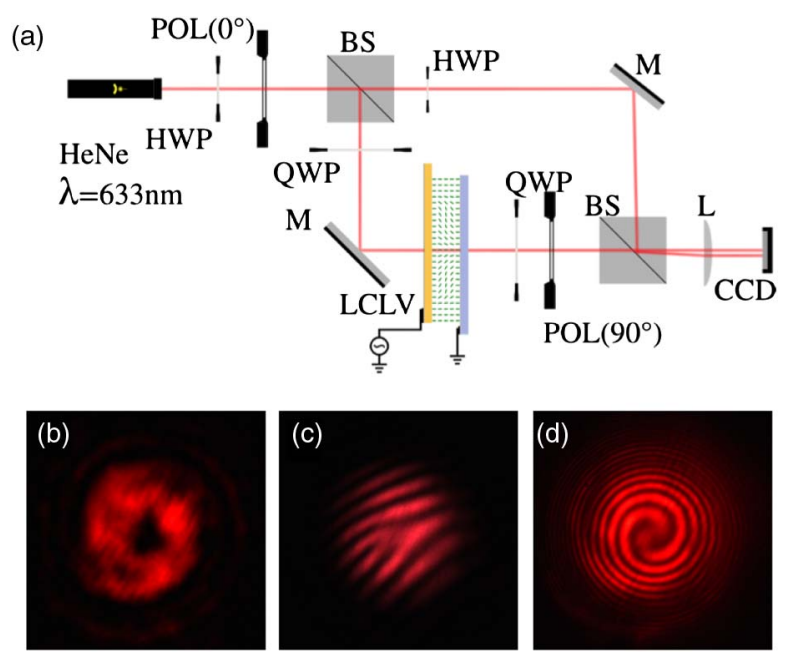

(a) Setup for the vortex induction in a LCLV and visualization of the optical wavefront with a Mach-Zehnder interferometer. POL, polarizer; QWP, quarter-wave plate; HWP, half-wave plate; Obj, microscope objective; M, mirror; BS, beam splitter; CCD, CMOS camera; L, lens; LCLV, liquid-crystal light valve. (b) Output intensity showing a Gauss-Laguerre beam, (c) fringe pattern after interference with a planar wavefront, and (d) spiraling interference patterns after interference with a spherical wavefront. Adapted from Fig. 2 in [45], copyright 2012, with permission from the American Physical Society.

To prove the vortex induction, a laser beam of wavelength $\lambda=632 \mathrm{~nm}$ and power $P=0.55 \mathrm{~mW}$ is focused to a diameter of $395 \mu \mathrm{m}$ on the photoconductive side of the LCLV [45]. The input beam polarization is taken either righthanded circular or left-handed circular. The bias voltage of the LCLV was fixed to $V_{0}=24 \mathrm{~V} \mathrm{rms}$ at frequency $100 \mathrm{~Hz}$. The results are displayed in Figs. 9(b)-9(d) for the RH input. The output beam exhibits a doughnut profile of the intensity [Fig. 9(b)]. A dislocation of charge 2 in the plane wavefront interference fringes [Fig. 9(c)] and a two-arm spiral for the spherical wavefront interference patterns [Fig. 9(d)] confirm that the output beam has a phase singularity with winding number 2 . The spin-to-orbital angular momentum transfer during the vortex induction is consistent with a +1 q-plate, confirming that the matter vortex is a +1 defect.

\section{Vortex Induction Provides Photo-Addressable q-Plates}

Due to the azimuthal profile of the molecular reorientation, the optically induced defect acts like a q-plate with $q=1,1$ being the topological charge of the defect induced in the nematic structure [46,72]. As represented in Fig. 10, the incoming circularly polarized field is converted to a beam with opposite polarization and with an additional helical phase factor exp $2 i q \xi$ through a conversion factor $\sin ^{2}(\Delta / 2)$. As a matter of fact, an incoming $\mathrm{LH} / \mathrm{RH}$ circularly polarized Gaussian input beam entering the LCLV will exit, for the ideal case $\Delta=\pi$, with $\mathrm{RH} / \mathrm{LH}$ circular polarization and an additional helical phase with winding number \pm 2 (in our case $q=1$ ). 


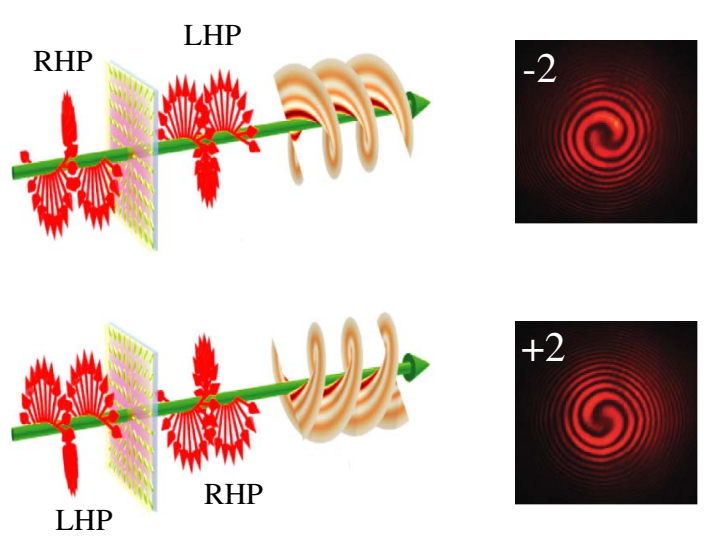

Schematic representation of the spin-orbital angular momentum transfer during the vortex induction process: an RHP (LHP) circularly polarized beam is converted into an LHP (RHP) beam with a phase singularity of topological charge $-2(+2)$; experimental interferograms are shown beside the respective panels. The induced $q=1$ plate is schematically depicted by the director orientation around the matter vortex. Adapted from Fig. 1 in [72].

The direction of the spiral arm rotation is reversed for the two types of polarization, thus confirming that, for the $\mathrm{LH} / \mathrm{RH}$ input, the output is $\mathrm{RH} / \mathrm{LH}$ polarized with topological charge \pm 2 .

\subsection{Induction of the Opposite Topological Charge}

Due to the geometrical configuration of the cells and the anisotropy of the liquidcrystal elastic constants, the optical vortex induction also induces a positive vortex, or umbilic with $2 \pi$ winding number. As seen above, this acts as a $q=+1$ plate. To induce the matter vortex with the opposite topological charge, hence a $q=-1$ plate, we can proceed in the following way. We induce two adjacent vortices with topological charge +1 and, by increasing the voltage applied to the LCLV, the deformation around each vortex reaches the adjacent one, thus creating a spontaneous vortex with the opposite topological charge between the two, to fulfill the constraints imposed by the continuity of the zero-level curves of the director field.

An example of vortex induction with -1 topological charge is shown in Fig. 11. For comparison, the upper panels display the induction of a +1 charged vortex. The equivalent q-plates lead to the generation of an optical vortex with charge $-2(+2)$ for the $+1(-1)$ vortex and left-handed circular polarization (LHP) of the input beams and to the generation of an optical vortex with charge $+2(-2)$ for the $+1(-1)$ vortex and right-handed circular polarization (RHP) of the input beams [46]. This process is illustrated by the experimental snapshots in Fig. 11.

Numerical simulations of the anisotropic and forced Ginzburg-Landau equation [Eq. (13)] exhibit similar behavior. When two +1 adjacent vortices are initially addressed, a -1 vortex spontaneously appears between them [46]. A numerical snapshot showing the generation of the -1 vortex between two positive vortices is shown in Fig. 12. This procedure can be exploited for the selective creation of \pm 1 topological charges of the photoinduced vortices. As we will show in 
(a)

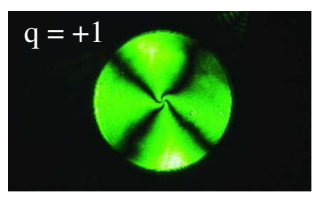

(b)

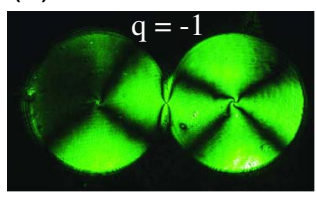

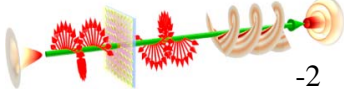
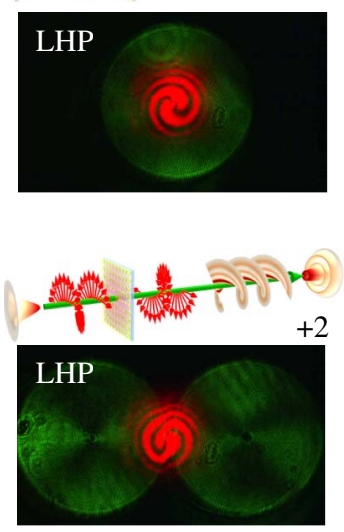
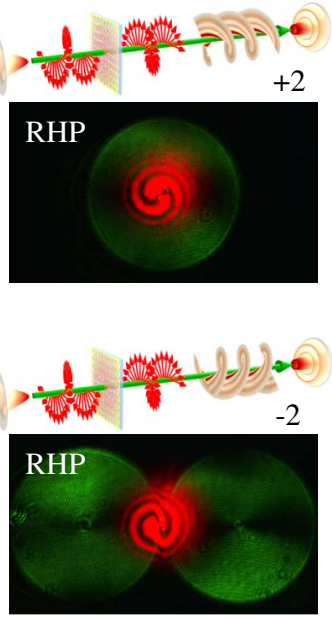

Experimental realization of the $q=+1$ vortex (upper panels) and $q=-1$ vortex (lower panels). Left panels: a $q=+1$ defect is created in the center of an illuminated area and a $q=-1$ defect is generated between two spots. Central panels: for an input LHP, the $q=+1(q=-1)$ defect yields an optical vortex with charge $-2(+2)$. Right panels: for an input RHP, the $q=+1(q=-1)$ defect produces an optical vortex with charge $+2(-2)$. Adapted from Fig. 5 in [46], copyright 2013, with permission from the American Physical Society.

\section{Figure 12}

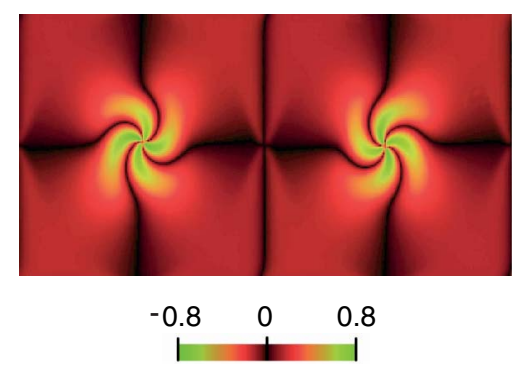

Nullcline field $\psi(\vec{r}, t)$ obtained from numerical simulation of the anisotropic forced Ginzburg-Landau equation [Eq. (13)] with periodical boundary conditions, forced with two localized Gaussian beams, on the left and right side, respectively. Two vortices of positive charge are initially addressed, a vortex with negative charge spontaneously appears between them. Adapted from Fig. 5 in [46], copyright 2013, with permission from the American Physical Society.

Section 8 , the same process leads to the generation of interconnected arrays of +1 and -1 vortices.

\section{Origin of the Vortex Induction Via Anisotropy Stabilized Light-Matter Interaction}

From the above experimental observations, it is clearly established that umbilics and vortex induction play important roles in the process of optical vortex generation. In the following section the role of the photoconductor is taken into 
account for the generalization of the theoretical description of defect induction in photosensitive liquid-crystal media. We will see how the inclusion of this element translates into an external forcing in the amplitude equation describing the director field deformation.

\subsection{Amplitude Equation Close to the Fréedericksz Transition for an LCLV}

Let us consider a nematic liquid-crystal layer with negative anisotropic dielectric constant $\varepsilon_{a}<0$ and homeotropic anchoring under the influence of lowfrequency voltage of the order of kilohertz. The orientation of the director is described by the vector field $\vec{n}(\vec{r}, t)$, where $\{\vec{r}, t\}$ are the space and time coordinates. The dynamical equation for the molecular director $\vec{n}$ is the Frank-Oseen mode, which reads [55]

$$
\begin{aligned}
\gamma \partial_{t} \vec{n}= & K_{3}\left[\nabla^{2} \vec{n}-\vec{n}\left(\vec{n} \cdot \nabla^{2} \vec{n}\right)\right] \\
& +\left(K_{3}-K_{1}\right)[\vec{n}(\vec{n} \cdot \vec{\nabla})(\vec{\nabla} \cdot \vec{n})-\vec{\nabla}(\vec{\nabla} \cdot \vec{n})] \\
& +\left(K_{2}-K_{3}\right)[2(\vec{n} \cdot \vec{\nabla} \times \vec{n})(\vec{n}(\vec{n} \cdot \vec{\nabla} \times \vec{n})-\vec{\nabla} \times \vec{n}) \\
& +\vec{n} \times \vec{\nabla}(\vec{n} \cdot \vec{\nabla} \times \vec{n})]+\varepsilon_{a}(\vec{n} \cdot \vec{E})[\vec{E}-\vec{n}(\vec{n} \cdot \vec{E})],
\end{aligned}
$$

where $\gamma$ is the rotational viscosity coefficient, $\varepsilon_{a}$ is the anisotropic dielectric constant that accounts for nonlinear response of the dielectric constant, and $\left\{K_{1}, K_{2}, K_{3}\right\}$ are the nematic liquid-crystal elastic constants, which account, respectively, for the elastic deformation of the splay, twist, and bend types. The electric field is given by $\vec{E}=(V / d) \hat{z} \equiv E_{z} \hat{z}$, where $E_{z}$ is the root mean square amplitude of the electric field, $V$ is the applied voltage, and $d$ is the width of the liquid-crystal layer.

A trivial equilibrium of the liquid-crystal layer consistent with the anchoring condition is the homeotropic state, $\vec{n}=\hat{z}$. To study the dynamics around this equilibrium, let us consider the ansatz $\vec{n}=\left(u, v, 1-\left(u^{2}+v^{2}\right) / 2\right)$, where $\{u(z, t), v(z, t)\}$ are small perturbations. Retaining the leading order, we obtain the following set of decoupled equations:

$$
\begin{aligned}
& \gamma \dot{u}=K_{3} \partial_{z z} u-\varepsilon_{a} E^{2} u, \\
& \gamma \dot{v}=K_{3} \partial_{z z} v-\varepsilon_{a} E^{2} v .
\end{aligned}
$$

By taking a solution of the form $u(z, t)=u_{0} e^{\sigma t} \sin (\pi n z / d)$ and $v(z, t)=$ $v_{0} e^{\sigma t} \sin (\pi n z / d)$, with $\left\{u_{0}, V_{0}\right\}$ constants and $n$ an integer number, we obtain the rate growth relation

$$
\gamma \sigma_{n}=-K_{3}\left(\frac{\pi n}{d}\right)^{2}-E^{2} \varepsilon_{a}
$$

Hence, the homeotropic state undergoes a degenerate stationary instability $(\sigma=0)$ when the anisotropic dielectric constant is negative $\left(\varepsilon_{a}<0\right)$ for critical values of the voltage that match the Fréedericksz transition threshold $V_{\mathrm{FT}}=$ $\sqrt{-K_{3} \pi^{2} / \varepsilon_{a}}$. Then, the director undergoes orientational instability, i.e., the molecules tend to align perpendicularly to the electric field $\vec{E}$. As a result of the elastic coupling between the molecules, the director has a cone of possible 
equilibria. From the point of view of bifurcation theory, this instability corresponds to a degenerate pitchfork bifurcation with $O(2)$-symmetry [69]. Figure 13 outlines the bifurcation diagram for this instability.

To figure out the mechanism of creation and pinning of matter vortices, we derive an amplitude equation in the vicinity of the Fréedericksz transition. Illuminating the LCLV with a Gaussian beam, induces a voltage drop with a bell-shaped profile across the liquid-crystal layer, higher in the middle of the illuminated area. To determine the shape of the voltage drop within the sample, one can consider the sample as consisting of two infinitely extended planar parallel plates separated by a thickness $d$. The upper plate, located in $z=d$, is lit by a Gaussian beam. By introducing cylindrical coordinates, the voltage $V(r, \theta, z)$ satisfies the Laplace equation:

$$
\partial_{z z} V+\frac{\varepsilon_{\perp}}{\varepsilon_{\|}} \nabla_{\perp}^{2} V=0
$$

where $\nabla_{\perp}^{2}$ stands for the transversal Laplacian operator in polar coordinates. The voltage satisfies the boundary conditions in the respective plates $V(r, \theta, z=$ $d)=V_{0}+\alpha I(r)$ and $V(r, \theta, z=0)=0$, with $(r, \theta)$ the polar coordinates in the plane where the origin of the coordinate system corresponds to the center of the beam and $\theta=0$ corresponds to the $x$ axis [see Fig. 7(b)]. $I(r)$ stands for the intensity of the Gaussian beam, $I(r)=I_{0} e^{-r^{2} / \omega^{2}}$, with $I_{0}$ the peak intensity, $\omega$ the beam waist, and $\alpha$ a phenomenological parameter that describes the linear response of the photoconductor. By using the Fourier transform in polar coordinates and solving the above equation with the corresponding boundary conditions, after straightforward calculations one obtains [46]

$$
V(z, r)=\frac{1}{2 \pi} \int_{-\infty}^{\infty} \mathrm{d} k e^{-i k \cdot r_{\perp}} \frac{\sinh \left(\sqrt{\frac{\varepsilon_{\perp}}{\varepsilon_{\|}}} k z\right)}{\sinh \left(\sqrt{\frac{\varepsilon_{\perp}}{\varepsilon_{\|}}} k d\right)}\left(\int_{0}^{\infty} \mathrm{d} r_{\perp}^{*} e^{i k \cdot r_{\perp}^{*}}\left[V_{0}+\alpha I\left(r_{\perp}^{*} / \omega\right)\right]\right) .
$$

This expression is an exact analytical solution; however, it is too intricate to infer results from. For the sake of simplicity, one can consider the limit of a Gaussian beam sufficiently flattened $(\omega \rightarrow \infty)$. This limit is consistent with experimental

\section{Figure 13}

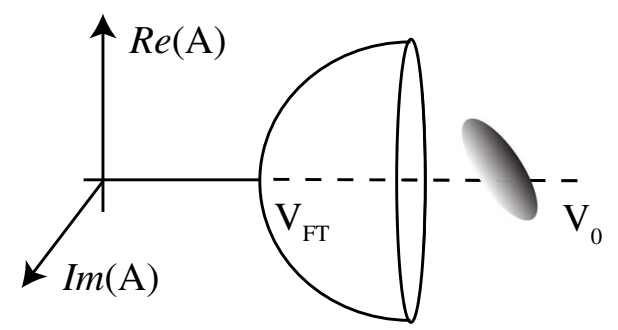

Schematic representation of Fréedericksz transition. The horizontal axis represents the voltage applied to the liquid-crystal sample. The transverse plane stands for the projection of the director in the horizontal plane of the sample. Adapted from Fig. 1 in [64], copyright 2014, with permission from the American Physical Society. 
observations, since the beam waist (about $200 \mu \mathrm{m}$ ) is much larger than the size of the vortex $(5 \mu \mathrm{m})$. In this limit the above expression, at the dominant order, takes the form

$$
V(z, r) \approx \frac{z}{d}\left[V_{0}+\alpha I(r / \omega)\right]
$$

The first and second terms on the right-hand side of the above expression account for the externally applied bias and the voltage drop induced by the Gaussian beam impinging on the sample, respectively. Figure 14(a) illustrates the voltage drop inside the LCLV in a vertical cross-sectional view. Then, the electric field $\vec{E}(r, \theta, z)$ inside the LCLV takes the form

$$
\vec{E}=-\nabla V=E_{z} \hat{z}+E_{r} \hat{r}=-\frac{1}{d}\left[V_{0}+\alpha I(r / \omega)\right] \hat{z}-\frac{z \alpha}{d \omega} \frac{d I(r \omega)}{d r} \hat{r},
$$

with $\hat{z}$ and $\hat{r}$ the unitary vectors in cylindrical coordinates [see Fig. 14(a)]. Note that the electric field contains an axis-symmetrical structure. This structure of the electric field projected onto the $x-y$ plane corresponds to a vortex with positive charge and zero phase, which is similar to the one shown in Fig. 30(d). This structure of the electric field will be responsible for inducing a matter vortex. The presence of an inhomogeneous electric field strongly modifies the dynamics

\section{Figure 14}

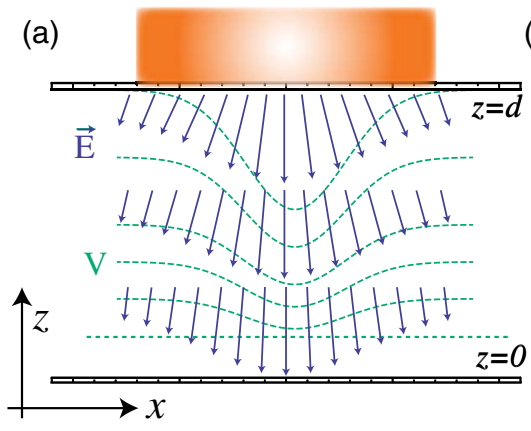

(b)
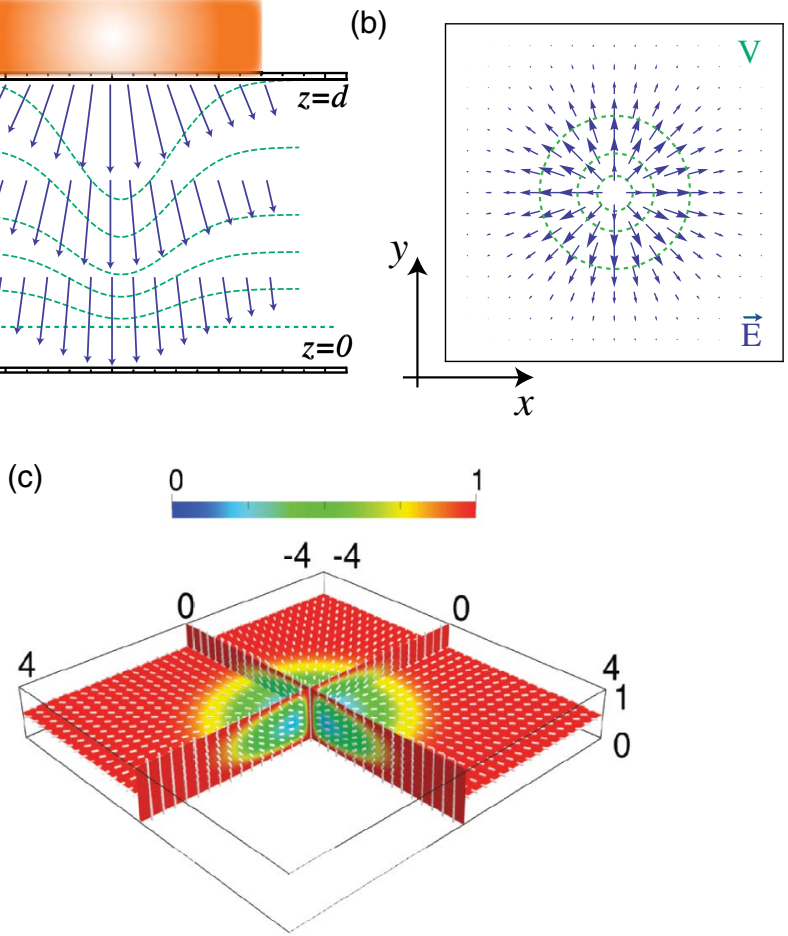

Electric field and voltage inside the liquid-crystal sample. (a) Vertical and (b) horizontal cross section showing a schematic representation of the electric field (arrows) and voltage drop (lines) across the liquid-crystal layer when a laser beam illuminates the sample from the top. The illuminating laser beam is impinging at the center of the sample. (c) 3D structure of the photoinduced umbilic. Adapted from Fig. 3 in [72]. 
of the liquid-crystal director $\vec{n}$. The dynamical equation for the molecular director $\vec{n}$ is given by Eq. (7). We consider the ansatz for the amplitude of the critical mode:

$$
\vec{n}(r, \theta, z) \approx\left(\begin{array}{c}
u(r, \theta, t) \sin \left(\frac{\pi z}{d}\right) \\
w(r, \theta, t) \sin \left(\frac{\pi z}{d}\right) \\
1-\frac{\left(u^{2}+w^{2}\right)}{2} \sin ^{2}\left(\frac{\pi z}{d}\right)
\end{array}\right) .
$$

Introducing the above ansatz in the director equation [Eq. (7)], integrating in the $z$ coordinate over one period, and by defining the complex amplitude $A \equiv u+i w$, after straightforward calculations one obtains (the anisotropic forced Ginzburg-Landau equation [46])

$$
\gamma \partial_{t} A=\mu A-a A|A|^{2}+\nabla_{\perp}^{2} A+\delta \partial_{\eta \eta} \bar{A}+b \frac{E_{r}(z)}{z} E_{z} e^{i \theta},
$$

which is the amplitude equation for self-stabilization of the matter vortex [46]. A similar procedure to derive the amplitude equation is presented in Appendix A. Here $\mu \equiv-K_{3} k^{2}-\varepsilon_{a} E_{z}^{2}(r, z)$ is the bifurcation parameter (note that $\mu$ is a spatially dependent parameter), $a \equiv-\left(-K_{1} k^{2} / 2+3 \varepsilon_{a} E_{z}^{2} / 4\right)>0$ is a parameter of order 1 that accounts for the nonlinear saturation, and $b \equiv \varepsilon_{a} 2 d / \pi$. The details of the derivation of the anisotropic forced Ginzburg-Landau equation will be presented in Appendix A.1.

The first expression does not depend on the $z$ coordinate and it is proportional to the derivative of a Gaussian. $E(z, r)$ corresponds to a Gaussian translated. Hence, the last term on the right-hand side of the anisotropic forced Ginzburg-Landau equation is an external forcing generated by the inhomogeneous radial electric field, which, in turn, is induced by the inhomogeneous profile of the light beam. This forcing term is responsible for inducing a matter vortex with positive charge in the center position where the applied Gaussian beam is peaked, which is at the origin of the self-stabilization mechanism for the vortex induction.

\subsection{Positive Vortex with Swirling Arms}

When the Fréedericksz transition starts from the unstable state $A=0$ in the presence of noise, or under uniform illumination, the system initially generates a large number of vortices that subsequently annihilate by pairs of opposite topological charge or fade toward the edges (see Figs. 15 and 16). Let us now consider the effect of forcing, $E_{r} \neq 0$, that occurs in the presence of a nonuniform illumination. Such a forcing simultaneously breaks the translational symmetry and the spatial rotation and, consequently, leads to a single positive vortex to be attracted and trapped in the central position of the illuminated area.

Figures 15 and 16 show sequences of pictures from, respectively, experimental observation and numerical simulations of the forced amplitude equation [Eq. (13)] without anisotropy $(\delta=0)$, illustrating the process described above. Starting from three generated vortices, a couple of them, oppositely charged, mutually attract and annihilate [see Figs. 16(a)-16(c)], thus leaving a single positive vortex at the end [Fig. 16(c)]. The single vortex is then attracted to the center of the illuminated area, where it remains pinned [Fig. 16(d)]. A similar dynamical evolution is observed in the experiment as it is depicted in Fig. 15. If we illuminate a large area of the LCLV, the system generates several vortices that 


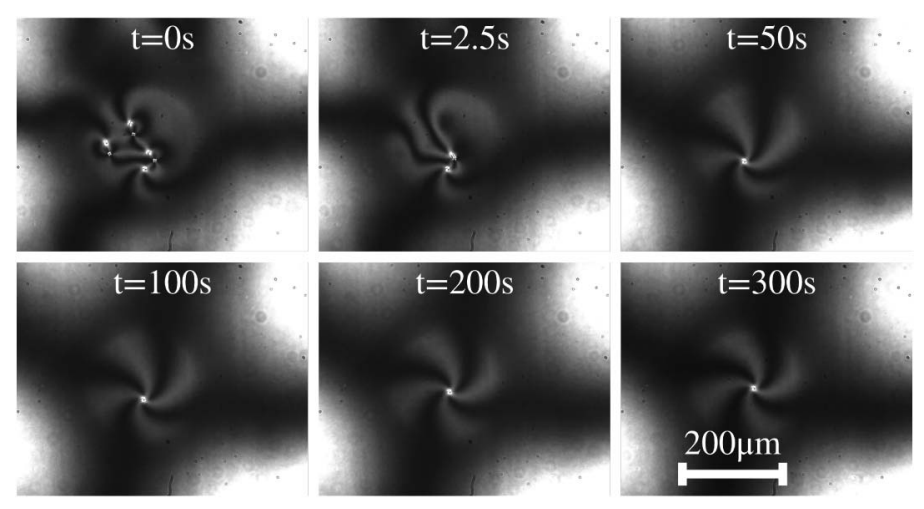

Temporal sequence of experimental observations of the dynamical evolution of vortices recorded with crossed polarizers.

subsequently annihilate by pairs of opposite topological charge, leaving just one vortex at the end.

Figures 17(e) and 17(f) show, respectively, the nullcline field and the corresponding phase of an initial unstable vortex, which is inconsistent with the forcing. This state evolves to a stable swirling vortex, as shown in Figs. 17(a) and 17(b). Note that the phase jump is always consistent in the outer region with that imposed by the forcing, while the inner region is consistent with the anisotropy. Notice also that negatively charged vortices are not consistent with the charge induced by the forcing; therefore, they are not a steady state.

We now consider simultaneously the effects of the elastic anisotropy, which is intrinsic to the medium properties, and the spatial forcing that is induced by the light. While the forcing induces a vortex that is pinned at the center of the light beam and tries to impose the phase jump, the elastic anisotropy imposes a phase jump that must be consistent with the four possible $\varphi_{0}$, which will be explained in the following sections. Because the anisotropy is proportional to the spatial derivatives, we expect it to be more relevant near the core of the vortex. Therefore, one expects that the anisotropy imposes the phase jump in the region close to the vortex core, and the spatial forcing imposes the phase jump in the

\section{Figure 16}

(a)

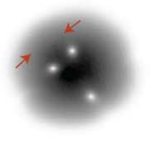

(b)

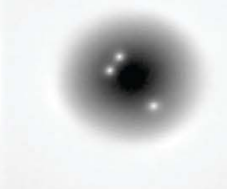

(c)

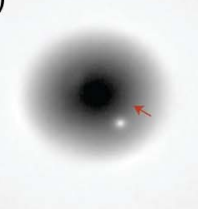

(d)

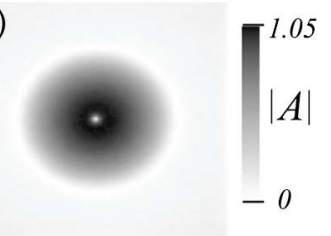

Dynamical evolution of the vortices observed in the forced amplitude equation [Eq. (13)] without anisotropy $(\delta=0)$ starting from the unstable state, $A=0$, with an initial noise. The temporal evolution is from the left to the right panel. The magnitude of the amplitude $|A|$ is displayed in gray. The steady state, which corresponds to a single vortex trapped in the center, is shown in the right image. Simulations were realized with $\mu=-0.5+1.69 e^{-r^{2} / \sigma^{2}}, \sigma=18, \delta=0.7$, and $b \frac{E_{r}}{z} E_{z}=0.00169 r e^{-r^{2} / \sigma^{2}}$. Adapted from Fig. 5 of [72]. 

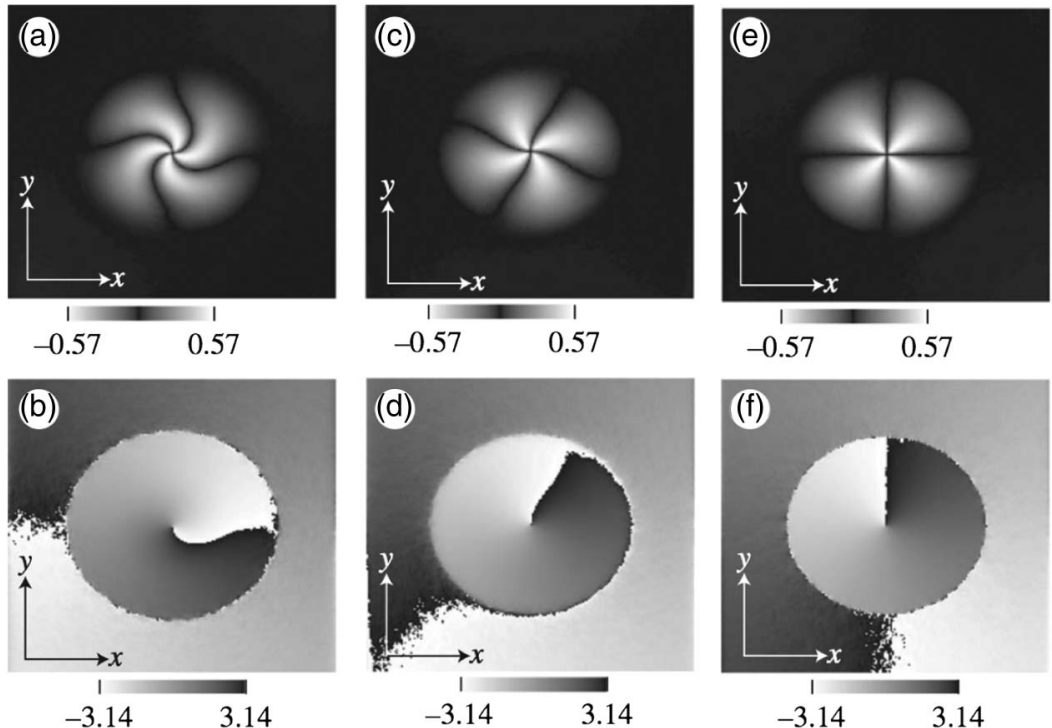

Nullcline and phase field, top and bottom panels, respectively, of vortex solutions of the anisotropic and forced amplitude equation [Eq. (13)] with positive anisotropy $(\delta>0)$, where the forcing term of Eq. (13) is replaced by $b\left[E_{r}(z) / z\right] E_{z} e^{i \theta+\varphi_{0}}$ and considering different $\varphi_{0}$ angles, respectively, $\varphi_{0}=\{0, \pi / 4, \pi / 2\} . \mu=-0.5+1.69 e^{-r^{2} / \sigma^{2}}, \sigma=18, \delta=0.7$, and $b \frac{E_{r}}{z} E_{z}=$ $0.00169 r e^{-r^{2} / \sigma^{2}}$. Adapted from Fig. 4 in [72].

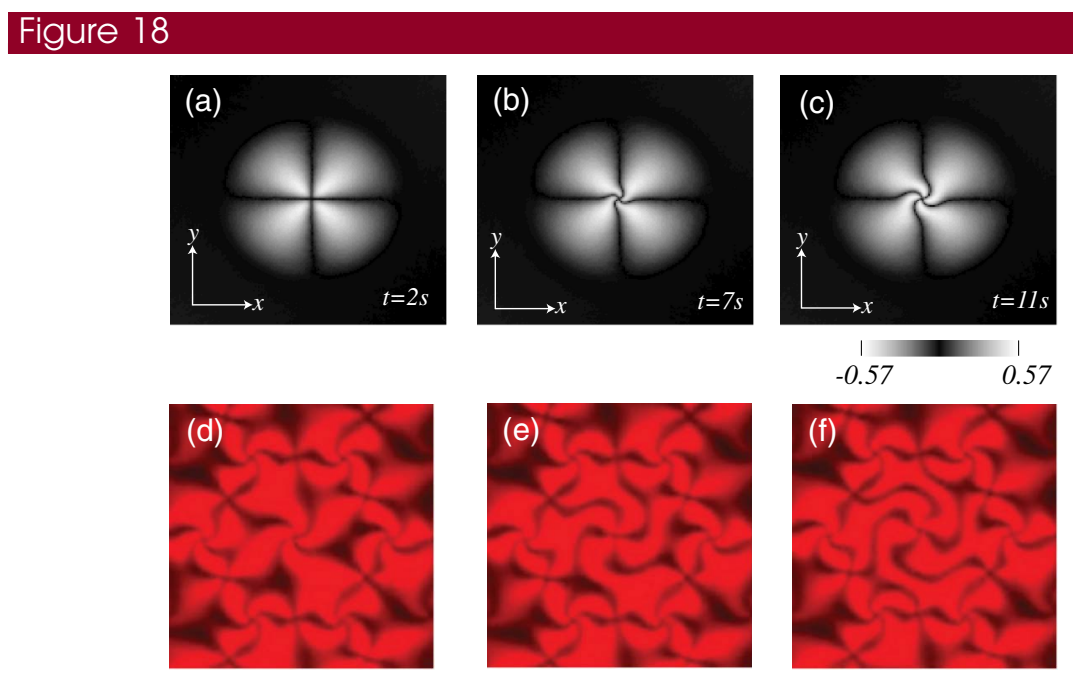

(a)-(c) Adapted from Fig. 6 in [72]. Bending process of the arms of a single positive vortex obtained from the forced amplitude equation [Eq. (13)] for positive elastic anisotropy. The time evolution proceeds from left to right. The nullcline field $\psi$ is displayed in gray. During its evolution, the vortex develops a swirling of the arms around its core. The final configuration is shown in (c). Simulations were realized with $\mu=-0.5+1.69 e^{-r^{2} / \sigma^{2}}, \sigma=18, \delta=0.7$, and $b \frac{E_{r}}{z} E_{z}=0.00169 r e^{-r^{2} / \sigma^{2}}$, and the time shown is scaled by $\gamma$. (d)-(f) Successive experimental snapshots (time delay $3.2 \mathrm{~s}$ ) recorded from the evolution of the vortex arms under geometrical frustration imposed from the boundaries. 
outer regions of the vortex. Figure 17 illustrates stationary vortices for positive anisotropy and with a forcing term of the form $b\left[E_{r}(z) / z\right] E_{z} e^{i \theta+\varphi_{0}}$, where $\varphi_{0}=$ $\{\pi / 2,3 \pi / 2\}$ are privileged [72]. in the left, center, and right panels are considered $0, \pi / 4$, and $\pi / 2$, respectively. As a result of adjusting the respective angles of the phase jump, the vortex solution exhibits a phase gradient, which induces a small force responsible for slightly displacing the vortex from its center.

Therefore, the origin of the bent arms of the vortices is, simply, the result of the competition between two effects: the anisotropy, imposing a phase jump angle in the core of the vortex, and the spatial forcing, imposing another phase jump angle in the outside regions. The top panels of Fig. 18 show the numerical evolution of a positive vortex initially consistent with the spatial forcing and its later temporal evolution resulting from the anisotropy. For comparison, a sequence of experimental snapshots showing similar evolution of the vortex arms is shown in the bottom panels of Fig. 18. To give more evidence to the swirling effect, the vortex was created in a position where a geometrical frustration was imposed by the presence of other vortices, which induces a large mismatch between the initial position of the vortex arms and the final equilibrium position fixed by the simultaneous occurrence of forcing and anisotropy.

\section{Vortex Arrays in Various Liquid-Crystal Systems}

\subsection{Torons in Photosensitive Frustrated Chiral Nematic Liquid Crystals}

Introduction of molecular chirality leads to chiral nematic liquid crystal (CNLC) phases, for which the ground-state director field $\vec{n}(\vec{r})$ shows a spatial twist, precessing at a constant rate along a helical axis. This constitutes a cholesteric phase, characterized by a helical arrangement of the molecular distribution [54-56]. The helix pitch can be controlled by changing the amount of chiral dopant in the nematic host. Under geometric confinement, the natural helix of the cholesterics can be unwound to form a uniform state. This uniform frustrated state can be twisted or untwisted back, with the possibility of forming twisted localized regions that behave like particle excitations immersed in a background field. The optical creation and multistable switching of localized configurations in the molecular orientation of a chiral nematic liquid crystal has been demonstrated in homeotropically aligned cells, where the confinement of the liquid-crystal layer induces a frustration of the helical structures [47].

In these experiments, the CNLC forms an equilibrium helical pitch $p$ confined into a cell of thickness $d$ and with the inner surfaces treated to induce homeotropic alignment of the molecules. These boundary conditions are incompatible with the ground-state cholesteric-phase twist, and tend to unwind the twisted structures, forcing a uniform bulk alignment with $\hat{n}$ orthogonal to the cell walls. The helical structure is unwound (nematic-like) when the cell gap is much smaller than the pitch $(p \gg d)$, whereas for larger cell gaps $(p \ll d)$, the director twists freely in the center of the sample and meets the vertical-boundary conditions at the glass plates by introducing splay and bend distortions. The CNLC unwinding occurs at $d / p \simeq 1$ or smaller, at which the sample is at first in a uniform initial state. Then, localized chiroelastic particle-like excitations-called triple-twist torons $(T 3 s)$-are generated by vortex laser beams and embed the localized 3D twist into a uniform background. 
To generate the torons, the CNLC cell is locally shone by Laguerre-Gauss vortex beams with helical wavefront and various topological charges, typically from $m=1$ to $m=10$. T3s are optically generated at will at a selected place and then structurally characterized with full detail in three dimensions. Confocal polarizing microscopy and computer simulations reveal the equilibrium internal structures of the torons, manifesting both skyrmion-like and Hopf fibration features [47]. The basic T3 configuration is a double-twist cylinder closed on itself in the form of a torus (Fig. 19(a)) and coupled to the surrounding uniform field by point or line topological singularities (Fig. 19(b)-19(f)). Remarkably, the torons enable twist in all three spatial directions and are stabilized by strong energy barriers associated with nucleation of topological defects.

Similar structures have been recently exploited for the realization of reconfigurable arrays of optical vortex generators [48]. These generators are elastic particle-like structures optically inscribed in frustrated cholesteric films by means of the laser-induced local winding of the chiral liquid-crystal mesophase. Such

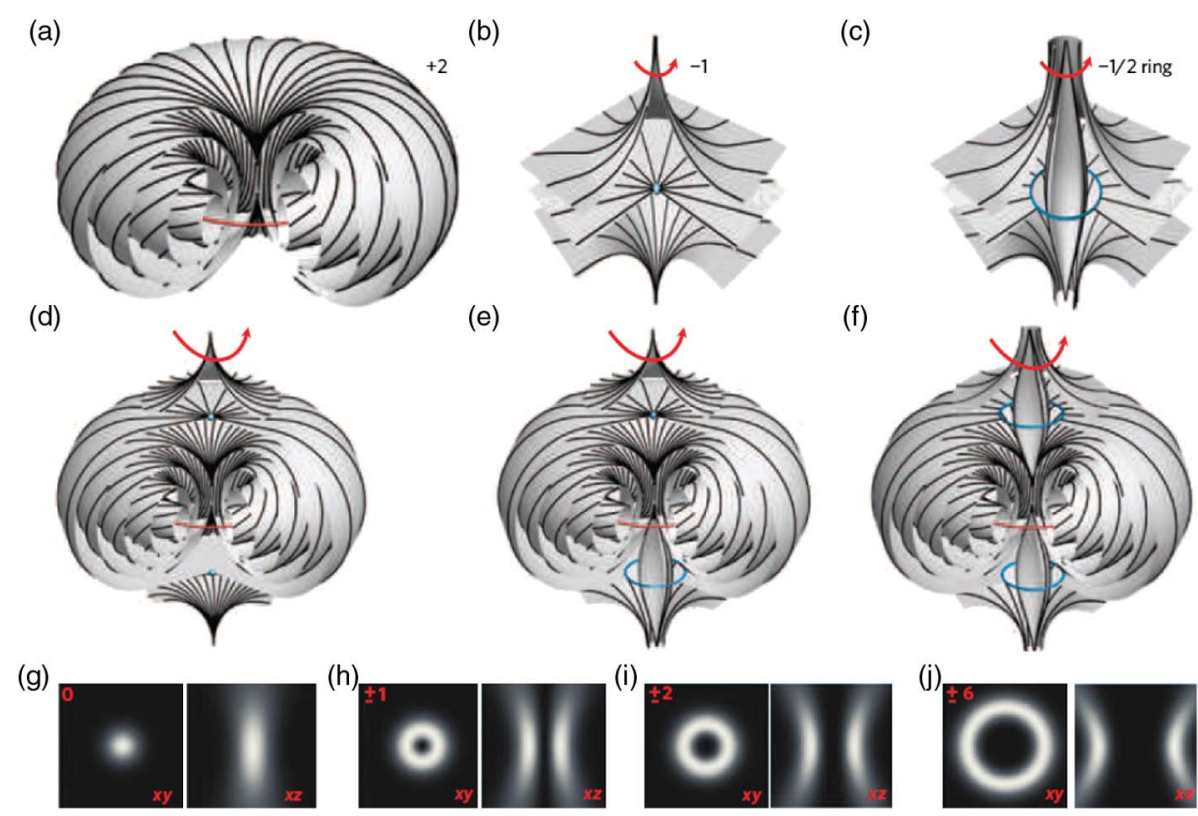

Triple-twist toron field configurations generated by Laguerre-Gauss beams and embedded into a uniform field by defects. Panel a shows a toron structure with topological charge +2 owing to the twist-escaped nonsingular disclination ring of strength $s=+1$ shown by the red line. Panels (b) and (c) show, respectively, a -1 hyperbolic point defect and a ring of $s=-1 / 2$ disclination topologically equivalent to a -1 point defect, both showing twist of $\vec{n}(\vec{r})$ with the sense of twist shown by the red arrows. Panel (d) is the T3-1 configuration with the toron accompanied by two hyperbolic point defects. Panel (e) shows a T3-2 structure containing a point defect and a disclination ring. Panel (f) is a T3-3 configuration with two $s=-1 / 2$ defect rings. Panels (g)-(j) show light-intensity distributions in the transverse $x-y$ (left) and axial $x-z$ (right) planes of the Laguerre-Gaussian beams of topological charge marked for each of the image pairs; the square cross sections are $4 \mu \mathrm{m}$ wide. Reprinted by permission from Macmillan Publishers Ltd: Smalyukh et al., Nat. Mater. 9, 139-145 (2010) [47]. Copyright 2010. 
(a)

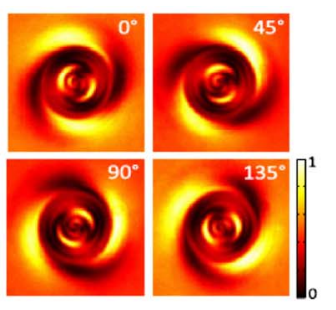

(b)

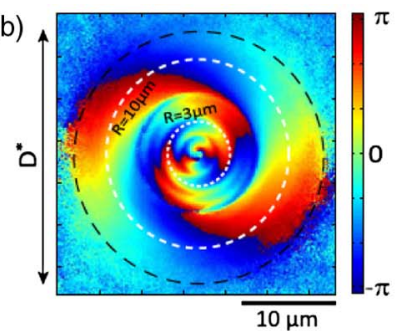

(c)

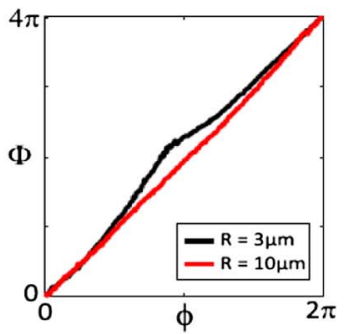

Optical vortex generation by optical addressing of frustrated chiral nematic films. (a) Normalized intensity spatial distribution $I_{\alpha}$ with $\alpha=0^{\circ}, 45^{\circ}, 90^{\circ}$, and $135^{\circ}$ under circularly polarized monochromatic illumination, where $I_{\alpha}$ refers to the intensity distribution obtained by placing a polarizer at an angle $\alpha$ from the $x$ axis at the output of the film. (b) Phase spatial distribution of the contracircularly polarized component of the output field with respect to the circular polarization state of the incident probe beam deduced from the data shown in panel (a). (c) Azimuthal phase profile along circles of different radii that reveal a phase singularity with topological charge 2 . Reprinted with permission from Yang and Brasselet, J. Opt. 15, 044021 (2013) [48].

structures are shown to produce optical vortices with a well-defined orbital state resulting from the spin-to-orbital angular momentum conversion process. The structure of the vortex generators is unveiled by polarimetric measurements, as shown in Fig. 20.

\subsection{Self-Assembled Defects in Smectic Liquid Crystals}

Recently, a method to produce closely packed ordered optical vortex arrays using self-assembled smectic liquid-crystal defects has been demonstrated [49-51]. These defects are called focal conic domains (FCDs) because of the conical symmetry of the director layers orienting tangentially along circular focal conical surfaces. The typical structure of a FCD is shown in Fig. 21(a).

FCDs in smectic layers organize spontaneously into vortex lattices with hexagonal symmetry. Each defect on the lattice sites has a size of the order of 10-35 $\mu \mathrm{m}$ and generates an optical vortex with topological charge $m=2$. The spiral profiles obtained in the interferometric observations have confirmed the formation of optical vortices, as predicted by the Jones matrix calculations. An example of

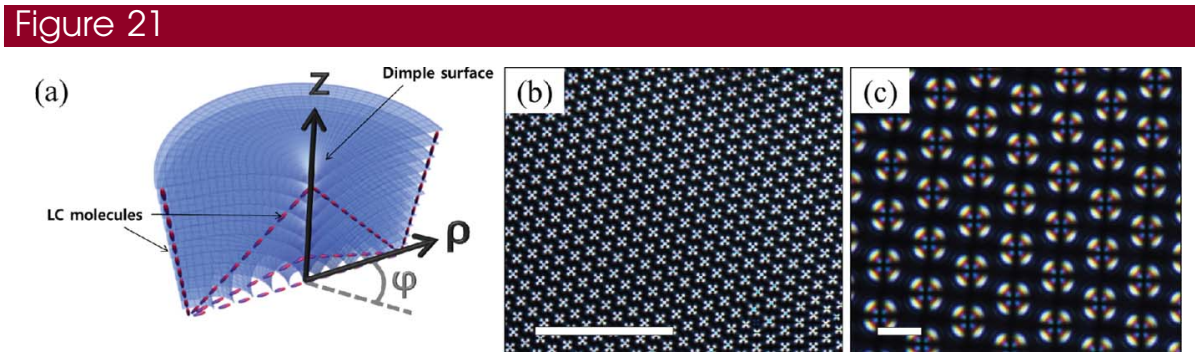

Experimental observation of the focal conic defects. (a) 3D view of the FCD in a smectic layer. (b) Image of the hexagonal FCD arrays between crossed polarizers; scale bar is $100 \mu \mathrm{m}$. (c) Magnified image; scale bar is $10 \mu \mathrm{m}$. Reprinted with permission from Son et al., Opt. Express 22, 4699 (2014) [51]. 
a self-assembled FCD vortex array is shown in Fig. 21(b). This method is very attractive because of its self-assembled nature and its ability to generate microscale vortices. Nevertheless, it must be considered that the arrays are fully connected, prohibiting the addressing of single vortex sites for a fully reconfigurable matrix.

\subsection{Umbilics in Nematics Driven by Microarrays of Electrodes}

Electrically tunable microarrays of q-plates have been recently realized and demonstrated to realize efficient conversion from spin-to-orbital angular momentum of an incoming light beam [52].

As shown in Fig. 22, the micropattern consists in a 1D array of $60 \mu \mathrm{m}$ side square electrodes. The liquid-crystal cell has a thickness of $13 \mu \mathrm{m}$ and is filled with a nematic liquid crystal with negative dielectric anisotropy. When driven with lowfrequency voltages a nonsingular topological defect, that is, an umbilical defect, is spontaneously formed in correspondence with each electrode patch.

The topological strength of the umbilic is found to be +1 in all cases. The typical intensity and phase distribution of the generated optical vortex with topological charge 2 depends on the geometrical factors of the patterned electrode as well as on the driving voltage. Even though the extension to $2 \mathrm{D}$ patterns seems not yet straightforward because of the increasing complexity of the electrical driving, the method is attractive in terms of potential applications for parallel processing of optical orbital angular momentum.

\subsection{Umbilics in Nematics Driven by Magnetic Fields}

The controlled generation of umbilics in a nematic layer with homeotropic anchoring has also been realized by using magnetic fields [53]. The method is an extension of that used previously by Gilli and co-workers for the study of Ising and Bloch walls in an almost homogeneous rotating magnetic field created by two parallelepipedic magnets [65]. In the paper by Pieranski et al. [53], the

\section{Figure 22}

(a)

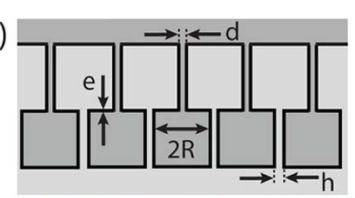

(b)

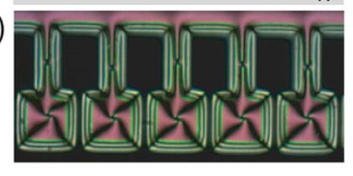

(c)

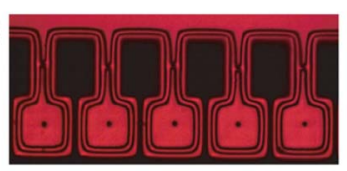

(d)

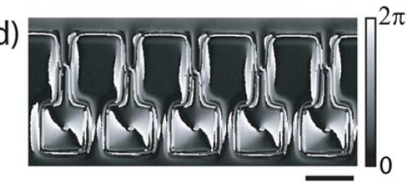

(a) Patterned electrode for the generation of umbilics. (b) Defects observed between crossed linear polarizers and under white-light illumination. (c) Defects observed between crossed circular polarizers and under $633 \mathrm{~nm}$ wavelength illumination. (d) Phase profile of the generated vortex field with topological charge 1; scale bar is $60 \mu \mathrm{m}$. Reprinted with permission from Loussert et al., Appl. Phys. Lett. 105, 121108 (2014) [52]. Copyright 2014, AIP Publishing LLC. 
nematic samples are submitted to magnetic fields created by small NdFeB magnets, and the induced umbilics are unveiled by observation between crossed polarizers in monochromatic or white light.

The sample consists of a nematic layer of thickness $d$ sandwiched between two glass plates coated with transparent ITO electrodes and homeotropically aligned. In its ground state without fields, the director field is homogeneous and the sample has axial symmetry around the $z$ axis. Owing to the anisotropy $\chi_{a}=\chi_{\|}-\chi_{\perp}$ of the diamagnetic susceptibility of the nematic liquid crystal, a magnetic field $\vec{B}$ applied to such a sample exerts a torque,

$$
\Gamma_{m}=\frac{\chi_{a}}{\mu_{0}}(\vec{n} \cdot \vec{B})(\vec{n} \times \vec{B}) \approx \frac{\chi_{a}}{\mu_{0}} B_{z}(\vec{n} \times \vec{B}),
$$

on the director and tilts it out from the initial orientation $\vec{n}=(0,0,1)$. The new orientation of the director results from the balance between this magnetic torque and the restoring elastic torque $\Gamma_{\mathrm{el}}$. In approximation of small distortions, $n_{x}$, $n_{y} \ll 1$ and $n_{z} \approx 1$, the elastic torque can be written as

$$
\Gamma_{\mathrm{el}}=K \frac{\partial^{2} \vec{n}_{\perp}}{\partial z^{2}} \times \vec{n}
$$

with

$$
\vec{n}_{\perp}(z)=\vec{n}_{\perp 0} \cos \left(\frac{\pi z}{d}\right)
$$

and one finally obtains

$$
\vec{n}_{\perp 0} \approx \frac{B_{z} \vec{B}_{\perp}}{B_{c}^{2}}
$$

where $B_{c}$ is the threshold of the Fréedericksz transition in magnetic field and $\vec{B}_{\perp}$ is the projection of the magnetic field on the $x-y$ plane of the sample. By using the above relationship, permanent magnets and magnetic fields suitable for the production of umbilics can be designed and realized.

Moreover, by adding an AC voltage to the sample and by using various geometrical distributions of magnets, umbilics of \pm 1 topological charge can be

\section{Figure 23}
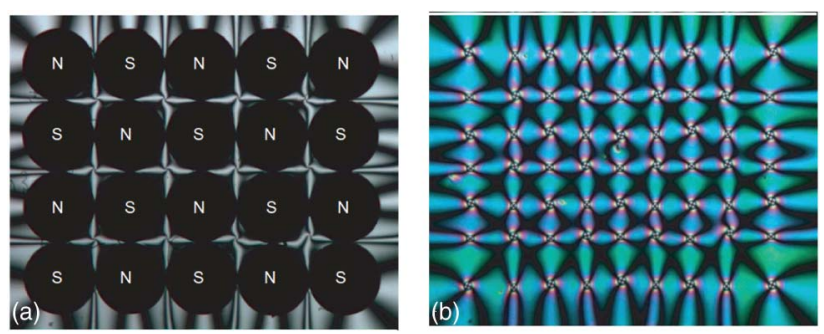

Generation of umbilics by a periodic system of magnets: panel a shows a $4 \times 5$ matrix made of magnets $2 \mathrm{~mm}$ in diameter, and panel $\mathrm{b}$ is the corresponding lattice of umbilics. [Copyright 2013 from "Generation of umbilics by magnets and flows," by Pieranski et al. [53]. Reproduced by permission of Taylor and Francis Group, LLC, a division of Informa.] 
selectively induced and arranged over the sample. In particular, a regular periodic system of magnets induces lattices of umbilics, as in the example reported in Fig. 23. Even though not yet exploited for photonic applications, such reconfigurable and magnetically driven arrays of vortices could constitute an additional possibility for the generation of topological charges via the interaction of light with soft matter systems.

\section{Programmable Optical Vortex Lattices in a Photo-Addressable Homeotropic LCLV}

The process of vortex induction via anisotropy stabilized light-matter interaction allows the creation of closely packed lattices of optical vortices with arbitrary and reconfigurable geometric distributions [46]. As long as liquid-crystal reorientation occurs only in the illuminated areas (which happens for relatively low amplitudes of voltage applied to the light valve), the vortices on adjacent lattice sites are independent from one another and all have the same +1 sign. Conversely, when reorientation occurs in the whole liquid-crystal layer (for large enough applied voltage), all the vortices become tightly coupled together, leading to the spontaneous generation of defects with -1 sign between adjacent lattice sites. The resulting vortex arrangements are consistent with the topologic conservation rules that accounts for the reconnection of reorientation lines in the distorted nematic layer.

Remarkably enough, all the topological reconnections in the light valve are reconfigurable, optically addressable, and tunable via the applied electric field. Moreover, the induced defect lattices act as arrays of photonic spin-to-orbital angular momentum couplers with both signs of the topological charge. For a circularly polarized input beam, the matter vortex at each lattice site generates a Laguerre-Gauss output beam with topological charge determined by the sign of the defect and the spin-to-orbital angular momentum transfer.

\subsection{Addressing Different Vortex Lattice Symmetries}

The setup for generating vortex lattices is sketched in Fig. 24. The beam of a diode-pumped frequency-doubled solid-state (DPSS) laser at wavelength $\lambda=532 \mathrm{~nm}$ is expanded, collimated, and directed to a spatial light modulator (SLM). The SLM is computer driven by intensity masks (an example is shown in the inset; the lattice period is $0.5 \mathrm{~mm}$, and the diameter of the vortex core is $1.2 \mu \mathrm{m}$ ), which, through a lens, are imaged onto the BSO side of the LCLV. The vortex beams at the LCLV output are recorded by a CCD camera. To observe the whole orientational structure inside the liquid-crystal layer, the LCLV can also be illuminated by white light and the transmitted field imaged at the CCD plane. Note that white light that illuminates the LCLV induces an extra voltage on the liquid-crystal sample. Therefore, the Fréedericksz threshold voltage with respect to the externally applied voltage $V_{0}$ decreases as a result of the additional voltage induced by the white light. Polarizers and red filters discriminate the green vortex beams from the white light transmitted through the valve. An He-Ne laser at wavelength $\lambda=632 \mathrm{~nm}$ is used to generate an interferometer, through which the phase singularities are visualized by making the whole vortex lattice interfere with an expanded collimated beam. 


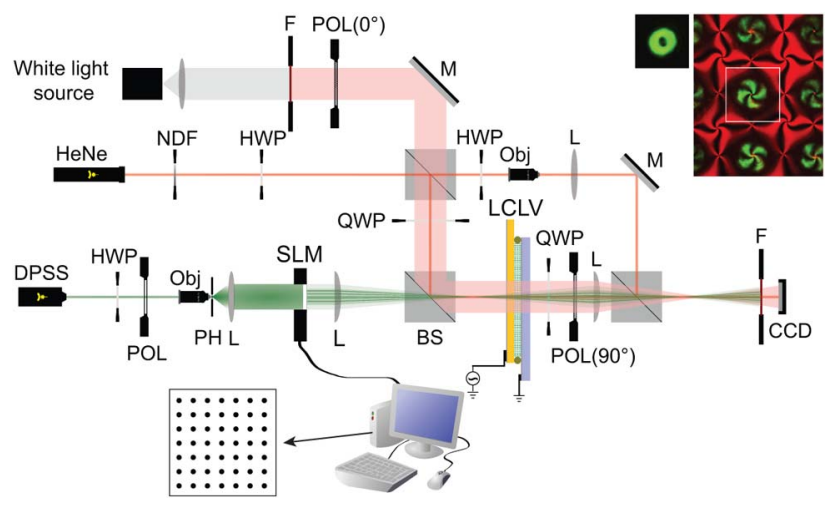

Experimental setup for optical writing of the vortex arrays. OBJ, objective; BS, beam splitter; M, mirror; SLM, spatial light modulator; NDF, neutral density filter; F, red filter; POL, polarizer; HWP, half-wave plate; QWP, quarter-wave plate; $\mathrm{PH}$, pinhole; L, lens; CCD, charge-coupled device camera. Bottom inset: example of a square modulation mask as input to the SLM. Upper inset: zoomedin view of the sample observed under crossed polarizers when illuminated with the square grid (green laser spots) and white light; on the left is an enlarged view of a single vortex. Reprinted with permission from Barboza et al., Phys. Rev. Lett. 111, 93902 (2013) [46]. Copyright 2013 American Physical Society.

\section{Figure 25}
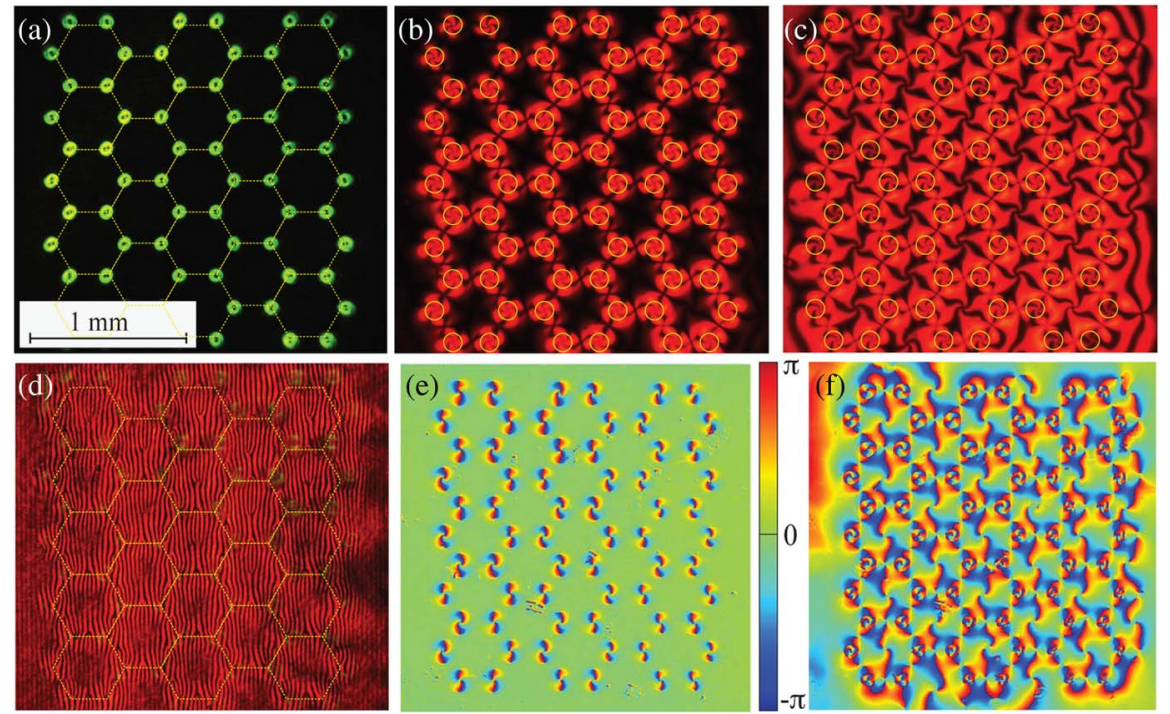

Hexagonal vortex lattices. (a) Laser intensity distribution, $V=19 \mathrm{~V}$; the dashed lines mark the lattice structure. (b) and (c) white-light images under crossed polarizers: (b) independent vortices, $V=18 \mathrm{~V}$, and (c) fully coupled vortex lattice, $V=22 \mathrm{~V}$; the circles indicate the positions of the addressing light spots. (d) Interferogram of the vortex lattice, $V=12 \mathrm{~V}$, with adequate lighting system to generate these diagrams; the input intensity is $I=250 \mu \mathrm{W} / \mathrm{cm}^{2}$. (e) and (f) Spatially resolved polarimetry: (e) $V=18 \mathrm{~V}$, and (f) $V=22 \mathrm{~V}$. [Adapted from Fig. 3 in [46], copyright 2013, with permission from the American Physical Society.] 
Defect lattices were generated with various symmetries and spatial distributions, specifically designing the intensity masks for the SLM to achieve close packing of the vortices. Examples of hexagonal vortex lattices are displayed in Fig. 25. At low applied voltage, the vortices are practically independent one from another and can be individually addressed [Figs. 25(a) and 25(b)]. Indeed, at low frustration, interactions between adjacent vortices can be neglected. When the voltage is increased, adjacent vortices become coupled through reorientation in the whole nematic background. Because of the topological constraints associated to the reconnection of reorientation lines, two (initially generated) adjacent vortices of equal sign induce a vortex of opposite charge in between them. An example of a fully connected network of vortices with alternating signs is visible in Fig. 25(c). The spatially resolved polarimetry of the vortex distribution in Figs. 25(e) and 25(f) immediately shows the sign of each vortex by the direction of circulation of the phase arms. Figure 25(d) presents the interferograms obtained with a plane reference wave. Note that the Freedericksz threshold voltage is here decreased due to the different illuminating conditions with respect to the situations of Figs. 25(a), 25(b), and 25(e). Moreover, the fork dislocation present in the middle of each group of six adjacent vortices (illuminated green spots) is a precursor of the matter vortex induced by topological constraints at higher voltage.

\section{Figure 26}

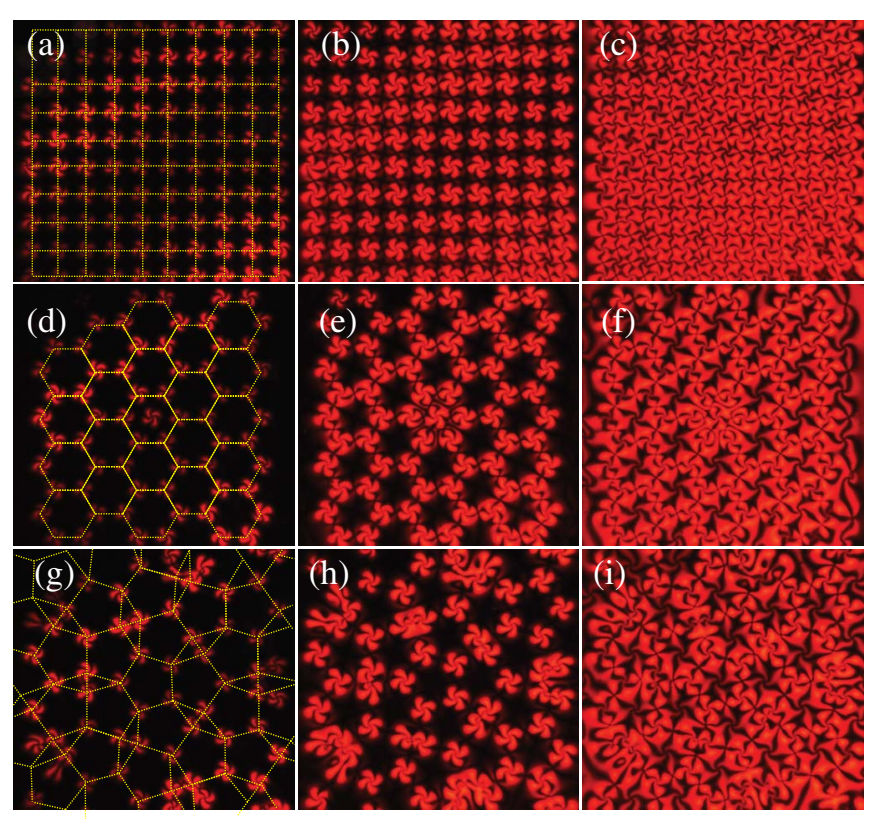

Vortex lattices with various spatial distributions; images are taken through crossed polarizers. (a)-(c) Squares with $V_{0}=14,18,22 V_{\text {rms }}$, (d)-(f) hexagons with a defect in the center for $V=14,18,22 V_{\mathrm{rms}}$, and (g)-(i) Penrose lattice for $V_{0}=14,18,22 V_{\mathrm{rms}}$; the dashed lines mark the corresponding lattice structures. The addressing light intensity is $I=250 \mu \mathrm{W} / \mathrm{cm}^{2}$ in all cases. [Adapted from Fig. 4 in [46] copyright 2013, with permission from the American Physical Society.] 


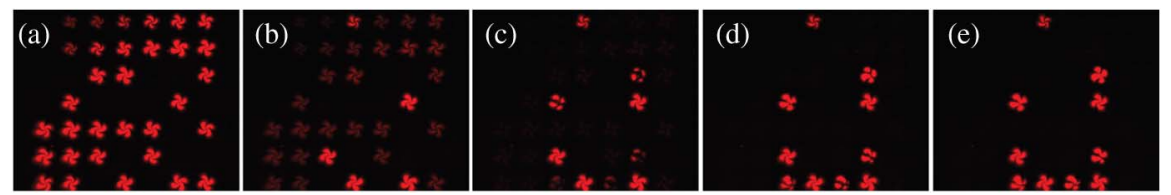

Dynamical transition between random arrays. The initial state in (a) is driven to the final state in (e) by addressing the light valve with another intensity mask. The experimental snapshots are separated by $250 \mathrm{~ms}$.

By employing suitably designed intensity masks, we created vortex lattices with various distributions, as shown in Fig. 26 for the cases of a square lattice, a Penrose lattice, and a hexagonal lattice with a topological frustration in the center. Again, as the bias was increased from low to high voltages, we observed the transition from independent vortices to a fully connected network of adjacent vortices of alternating signs. In hexagonal lattices, a topological frustration can be induced by addressing a defect in the center of a hexagonal cell. If this is done when the fully connected network is already established, the optically addressed defect undergoes a topological frustration with respect to the defect that was present at the same site. As a consequence, the unmatched reorientation lines reorganize themselves and give rise to a transient unwinding dynamic of the defect spiral arms, until the system is able to self-heal into a stationary configurational trade-off [46].

\subsection{Random Lattices: Dynamics of the Vortex Realignment}

Random lattices can be generated at will by appropriate intensity masks delivered by the SLM. An example showing the transition from a random lattice to another one is shown in Fig. 27, where the dynamical transition between the two can be appreciated. The switching time between the initial and the final configuration is approximately $1 \mathrm{~s}$.

\section{Conclusions and Outlook}

Liquid-crystal media provide versatile and efficient platforms for optical vortex generation. Either single selected vortices or fully connected vortex arrays can be addressed, depending on the specifically chosen liquid-crystal system, as well as on the method used to drive it. Liquid crystals exist in various mesophases and, correspondingly, the optical vortex generators consist of different structures embedded in the matter texture. One of the most widely used liquid-crystal matter templates capable of generating optical vortices is the umbilic, that is, a non-singular defect, string-like object in three dimensions, arising in nematic liquid crystals because of surface interactions [40], electric bias [41], or magnetic field driving [53]. Cholesteric liquid crystals, characterized by a chiral twist of the molecular arrangement, have been shown to produce, under appropriate frustrating confinement conditions, another type of optical vortex generator, the so-called toron, which is a chiral particle-like elastic structure where the twist is reestablished locally over a uniform background [47]. 
Another type of optical vortex generator is the FCD that arises in smectic liquid crystals [49].

Therefore, a large number of possibilities is offered by liquid crystals for optical vortex generation, and a growing interest for this domain of research is triggered by the high level of technological maturity reached by numerous liquid-crystal-based technologies. In particular, by using a nematic liquid crystal in a homeotropic light-valve geometry, we experimentally demonstrated a robust phenomenon of controlled vortex induction, which is, at the same time, spontaneous, self-stabilizing, and positionally stable. After its induction, the vortex develops a swirling of the arms, which remain stationary and stable. The process is described by a theoretical model based on an amplitude equation derived close to the Fréedericksz transition, which allows us to understand the origin of the induced vortex and the competition between the forcing induced by the light and the elastic anisotropy that generates the swirling arms of the vortex.

By using suitable intensity masks on the photosensitive wall of the light valve, reconfigurable and programmable vortex arrays can be induced and manipulated with a large degree of flexibility of the configurations. Interlaced lattices of +1 and -1 vortices are generated by exploiting the topological reconnection lines of the distorted nematic director field. In future work, methods for the selective generation of higher topological charges could be devised, either based on other geometrical configurations of liquid-crystal cells or the combined use of light valves with other liquid-crystal mesophases.

\section{Appendix A: Derivation of the Model Description}

\section{A.1. Derivation of the Amplitude Equation Close to the Fréedericksz Transition}

Let us consider a nematic liquid-crystal layer with negative anisotropic dielectric constant and homeotropic anchoring under the influence of low-frequency voltage of the order of kilohertz. For the sake of simplicity we neglect here the contribution of the transverse electric field. To understand the nonlinear response of the system at the onset of the Fréedericksz transition, we follow the weakly nonlinear analysis presented in Refs. [65,66]. Let us introduce

$$
\vec{n}(\vec{r}, t)=\left(\begin{array}{c}
n_{1}(\vec{r}, t) \\
n_{2}(\vec{r}, t) \\
1-\frac{n_{1}^{2}+n_{2}^{2}}{2}
\end{array}\right)
$$

into the Frank-Oseen equation:

$$
\begin{aligned}
\gamma \partial_{t} \vec{n}= & K_{3}\left[\nabla^{2} \vec{n}-\vec{n}\left(\vec{n} \cdot \nabla^{2} \vec{n}\right)\right] \\
& +\left(K_{3}-K_{1}\right)[\vec{n}(\vec{n} \cdot \vec{\nabla})(\vec{\nabla} \cdot \vec{n})-\vec{\nabla}(\vec{\nabla} \cdot \vec{n})] \\
& +\left(K_{2}-K_{3}\right)[2(\vec{n} \cdot \vec{\nabla} \times \vec{n})(\vec{n}(\vec{n} \cdot \vec{\nabla} \times \vec{n})-\vec{\nabla} \times \vec{n}) \\
& +\vec{n} \times \vec{\nabla}(\vec{n} \cdot \vec{\nabla} \times \vec{n})]+\varepsilon_{a}(\vec{n} \cdot \vec{E})[\vec{E}-\vec{n}(\vec{n} \cdot \vec{E})],
\end{aligned}
$$

and, by neglecting higher nonlinear orders, we obtain 


$$
\begin{aligned}
\gamma \dot{n}_{1}= & K_{3}\left[\nabla^{2} n_{1}+n_{1}\left(\left(\partial_{z} n_{1}\right)^{2}+\left(\partial_{z} n_{2}\right)^{2}\right)\right] \\
& -\left(K_{3}-K_{1}\right)\left[n_{1} \partial_{z z}\left(n_{1}^{2}+n_{2}^{2}\right) / 2+\partial_{x x} n_{1}+\partial_{x y} n_{2}\right] \\
& +\left(K_{2}-K_{3}\right)\left[-\partial_{x y} n_{2}+\partial_{y y} n_{1}\right]-\varepsilon_{a} n_{1} E_{z}^{2}\left(1-n_{1}^{2}-n_{2}^{2}\right), \\
\gamma \dot{n}_{2}= & K_{3}\left[\nabla^{2} n_{2}+n_{2}\left(\left(\partial_{z} n_{1}\right)^{2}+\left(\partial_{z} n_{2}\right)^{2}\right)\right] \\
& -\left(K_{3}-K_{1}\right)\left[n_{2} \partial_{z z}\left(n_{1}^{2}+n_{2}^{2}\right) / 2+\partial_{x y} n_{1}+\partial_{y y} n_{2}\right] \\
& +\left(K_{2}-K_{3}\right)\left[\partial_{x x} n_{2}-\partial_{x y} n_{1}\right]-\varepsilon_{a} n_{2} E_{z}^{2}\left(1-n_{1}^{2}-n_{2}^{2}\right) .
\end{aligned}
$$

The other remaining equation is trivially satisfied by the normalization condition. To take into account the dynamics of the critical mode, we consider the ansatz $\quad n_{1}=X(x, y, t) \sin \left(k_{c} z\right)+W_{1}(\vec{r}, t) \quad$ and $\quad n_{2}=Y(x, y, t) \sin \left(k_{c} z\right)+$ $W_{2}(\vec{r}, t)$, with $k_{c} \equiv \pi / d . \vec{W}=\left(W_{1}, W_{2}\right)$ stands for higher order corrections. Using this ansatz in the above set of equations and linearizing in $\vec{W}$, it reads

$\gamma \dot{X} \sin (k z)$

$$
\begin{aligned}
= & \left(K_{3} \partial_{z z}-\varepsilon_{a} E_{z}^{2}\right) W_{1}+K_{3} \sin (k z)\left[\nabla_{\perp} X-k^{2} X+k^{2} X\left(X^{2}+Y^{2}\right) \cos ^{2}(k z)\right] \\
& -\left(K_{3}-K_{1}\right) \sin (k z)\left[X k^{2}\left(X^{2}+Y^{2}\right)\left(\cos ^{2}(k z)-\sin ^{2}(k z)\right)+\partial_{x x} X+\partial_{x y} Y\right] \\
& +\left(K_{2}-K_{3}\right) \sin (k z)\left[\partial_{y y} X-\partial_{x y} Y\right]-\varepsilon_{a} X \sin (k z) E_{z}^{2}\left(1-\left(X^{2}+Y^{2}\right) \sin ^{2}(k z)\right),
\end{aligned}
$$

$\gamma \dot{Y} \sin (k z)$

$$
\begin{aligned}
= & \left(K_{3} \partial_{z z}-\varepsilon_{a} E_{z}^{2}\right) W_{2}+K_{3} \sin (k z)\left[\nabla_{\perp} Y-k^{2} Y+k^{2} Y\left(X^{2}+Y^{2}\right) \cos ^{2}(k z)\right] \\
& -\left(K_{3}-K_{1}\right) \sin (k z)\left[Y k^{2}\left(X^{2}+Y^{2}\right)\left(\cos ^{2}(k z)-\sin ^{2}(k z)\right)+\partial_{x y} X+\partial_{y y} Y\right] \\
& +\left(K_{2}-K_{3}\right) \sin (k z)\left[\partial_{x x} Y-\partial_{x y} X\right] \\
& -\varepsilon_{a} Y \sin (k z) E_{z}^{2}\left(1-\left(X^{2}+Y^{2}\right) \sin ^{2}(k z)\right) .
\end{aligned}
$$

The linear operator acting on $\vec{W}$ is

$$
\mathcal{L}=\left(\begin{array}{cc}
K_{3} \partial_{z z}-\varepsilon_{a} E_{z}^{2} & 0 \\
0 & K_{3} \partial_{z z}-\varepsilon_{a} E_{z}^{2}
\end{array}\right) .
$$

By introducing the inner product $\langle f \mid g\rangle=\int_{0}^{d} \mathrm{~d} z f \cdot g$, this operator is self-adjoint and its kernel is $\operatorname{Ker}\left\{\mathcal{L}^{\dagger}\right\}=\{(\sin (k z), 0),(0, \sin (k z))\}$. Hence, for solving the above linear equation, we apply the solvability condition or the Freedholm alternative, and we obtain

$$
\begin{aligned}
\gamma \dot{X}= & K_{3}\left[\nabla_{\perp} X-k^{2} X+k^{2} X\left(X^{2}+Y^{2}\right) / 4\right] \\
& -\left(K_{3}-K_{1}\right)\left[\left(\partial_{x x} X+\partial_{x y} Y\right)-X k^{2}\left(X^{2}+Y^{2}\right) / 2\right]+\left(K_{2}-K_{3}\right)\left[\partial_{y y} X-\partial_{x y} Y\right] \\
& -\varepsilon_{a} X E_{z}^{2}\left(1-3\left(X^{2}+Y^{2}\right) / 4\right), \\
\gamma \dot{Y}= & K_{3}\left[\nabla_{\perp} Y-k^{2} Y+k^{2} Y\left(X^{2}+Y^{2}\right) / 4\right] \\
& -\left(K_{3}-K_{1}\right)\left[\left(\partial_{x y} X+\partial_{y y} Y\right)-Y k^{2}\left(X^{2}+Y^{2}\right) / 2\right]+\left(K_{2}-K_{3}\right)\left[\partial_{x x} Y-\partial_{x y} X\right] \\
& -\varepsilon_{a} Y E_{z}^{2}\left(1-3\left(X^{2}+Y^{2}\right) / 4\right) .
\end{aligned}
$$

Introducing the change of variable $A(x, y, t) \equiv(X+i Y) / \sqrt{a}$; defining $\partial_{\eta} \equiv \partial_{x}+i \partial_{y}, \nabla^{2}=\partial_{\eta \bar{\eta}}, \mu \equiv-K_{3} k^{2}-\varepsilon_{a} E_{z}^{2}$, and $a=\left(2 K_{1} k^{2}-3 \varepsilon_{a} E_{z}^{2}\right) / 4$; and scaling the space by a factor $\sqrt{\left(K_{1}+K_{2}\right) / 2}$, we get $[65,66]$ 


$$
\partial_{t} A=\mu A-A|A|^{2}+\nabla^{2} A+\delta \partial_{\eta \eta} \bar{A},
$$

where $\delta=\left(K_{1}-K_{2}\right) /\left(K_{1}+K_{2}\right)$. This is the anisotropic Ginzburg-Landau equation, where $\mu$ is the bifurcation parameter, which accounts for the competition between the elastic and electric forces; the second term corresponds to nonlinear saturation; the third one accounts for the diffusion, which is a consequence of the elastic coupling; and the last term is the anisotropic correction, a consequence of how the different deformations in the system have different energy. Without this anisotropic term, $\delta=0$, this equation corresponds to the Ginzburg-Landau equation with real coefficients, an equation widely studied because of its importance in dynamical systems, as we have mentioned before.

Similar equations were derived before using the method of homogenization for a nematic liquid-crystal cell in the limit where its thickness approaches zero [64], and for modeling self-organization in an array of microtubules interacting via molecular motors in [74].

Note that Eq. (A3) can be rewritten in the form

$$
\partial_{t} A=-\frac{\delta \mathcal{E}}{\delta \bar{A}}
$$

where the free energy has the form

$$
\mathcal{E}(A, \delta) \equiv \int_{\Omega} \mathrm{d} S\left[|\nabla A|^{2}+\frac{1}{2}\left(\mu-|A|^{2}\right)^{2}+\delta \operatorname{Re}\left\{\left(\partial_{\eta} \bar{A}\right)^{2}\right\}\right]
$$

where $\Omega$ is a bounded domain. In other words, the time-dependent anisotropic Ginzburg-Landau equation [Eq. (A3)] is simply a gradient flow of the free energy. $\mathcal{E}$ is a Lyapunov functional. Therefore, the trivial equilibria that minimize the free energy are $|A|^{2}=\mu$. However, this equation has nontrivial inhomogeneous equilibria, as we will see in the following section.

\section{A.2. Isotropic Limit, the Ginzburg-Landau Equation with Real Coefficients}

Considering the isotropic limit $\left(K_{1}=K_{2}=K_{3}\right) \delta=0$, the above model reduces to the well-known CGLE with real coefficients:

$$
\partial_{t} A=\mu A-|A|^{2} A+\nabla_{\perp}^{2} A .
$$

This model with complex coefficients was initially proposed by Ginzburg and Landau for understanding superconductivity [75]. The Ginzburg-Landau equation has attracted much interest by describing several physical systems such as fluids, superfluids, superconductors, liquid crystals, magnetic media, and optical cavities $[7,76]$.

The Ginzburg-Landau model has a family of uniform solutions parameterized by the phase of the form $A=\sqrt{\mu} e^{i \phi_{o}}$, where $\phi_{o}$ is a constant. The connections among different domains of these solutions are separated by vortices. Hence, this equation admits stable dissipative vortex solutions with topological charge $\pm 1[7,68]$. 
Figure 28 illustrates the vortex solution of the Ginzburg-Landau equation. If one considers polar representation $A=R_{v}(r) e^{i\left(m \varphi+\varphi_{0}\right)}$, where $m= \pm 1$ is the topological charge, $\{r, \varphi\}$ are the polar coordinates in the plane, and $\varphi_{0}$ is a continuous parameter that accounts for the phase invariance of the amplitude equation [Eq. (5)], the modulus of the amplitude, $R_{v}(r)$, satisfies

$$
\mu R_{v}-R_{v}^{3}-\frac{m^{2}}{r^{2}} R_{v}+\frac{1}{r} \frac{d R_{v}}{d r}+\frac{d^{2} R_{v}}{d^{2} r}=0
$$

There are no analytical expressions for the defect solutions of this model, which were first observed numerically and characterized their asymptotic behavior in Refs. [77-79]. The asymptotic behavior is

$$
R_{v}(r) \rightarrow \begin{cases}r & r \rightarrow 0 \\ \sqrt{\mu}\left(1-\frac{1}{2 r^{2}}\right) & r \rightarrow \infty\end{cases}
$$

By using the Padé approximant, one can obtain suitable approximations for the vortex solutions [7]. Note that the equation for the modulus of the amplitude [Eq. (A.7) does not depend on the sign of the topological charge. Hence, both vortices are indistinguishable from the point of view of the magnitude of the amplitude and nullcline field. A suitable scalar field to characterize the dynamics of vortices both experimental and theoretical is the nullcline field $\psi(\vec{r}, t)=$ $\operatorname{Re}(A) \operatorname{Im}(A)$. This auxiliary field becomes zero when the real or imaginary part of $A$ vanishes. Then, the arms and position of the vortex are represented, respectively, by the zero and the intersection of the zero-level curves. The nullcline field for the vortex solutions takes the form $\psi(r, \varphi)=R_{v}^{2}(r) \sin \left(2\left(m \varphi+\varphi_{0}\right)\right) / 2$. Thus, the vortex arms are characterized by being straight and orthogonal, forming a cross whose center determines the position of the vortex. An example of this case is shown in Figs. 28(c) and 28(f). In addition, the phase jump is

\section{Figure 28}
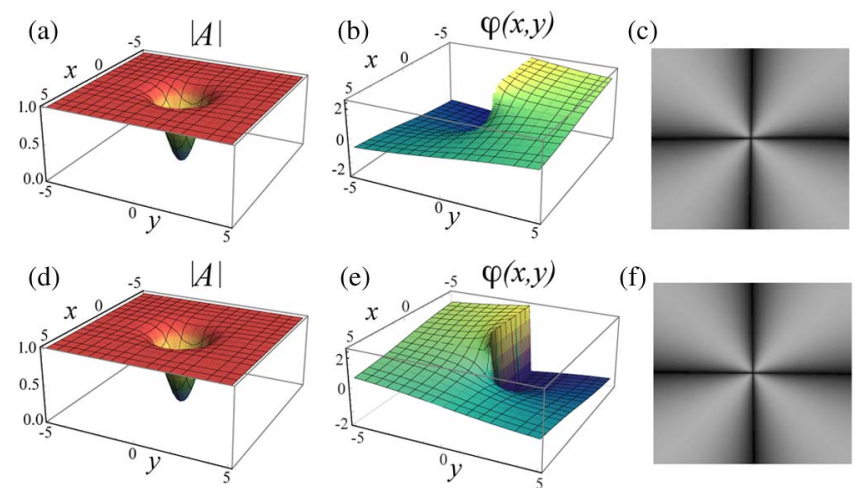

Vortex solutions of the Ginzburg-Landau equation [Eq. (5)] with $\mu=1$ (from numerical simulations using the finite difference method). Graphs of the magnitude (a), phase (b), and nullcline scalar field (c) of the positive vortex. Graphs of the magnitude (d), phase (e), and nullcline scalar field (f) of the negative vortex. [Adapted from Fig. 2 in [64], copyright 2014, with permission from the American Physical Society.] 
characterized by a straight line emerging from the position of the vortex [see Figs. 28(b) and 28(e)].

There is extensive literature devoted to the rigorous study of vortices in the complex Ginzburg-Landau equation (see [80] and references therein). The isotropic Ginzburg-Landau equation [Eq. (A6)] is invariant under the following symmetries: $\vec{r} \rightarrow \vec{r}+\vec{r}_{0}$ (spatial translation invariance), $\varphi \rightarrow \varphi+\varphi_{0}$ (coordinates rotation), $\varphi \rightarrow-\varphi$ (coordinates reflection), $A \rightarrow A e^{i \varphi_{0}}$ (phase invariance), and $A \rightarrow \bar{A}$ (reflection invariance).

\section{A.3. Anisotropic Ginzburg-Landau Equation with Real Coefficients: on the Nature of the Positive and Negative Vortices}

The equation for the amplitude magnitude does not depend on the sign of the topological charge. Hence, the two vortices are indistinguishable from the point of view of their magnitude and nullcline field. Figure 28(c) shows the nullcline field obtained by using the Ginzburg-Landau equation [Eq. (5)]. Note that the two defects are still indistinguishable (see Fig. 28); however, these defects are experimentally distinguishable (see in the inset of Fig. 5) [60,81,82]. The different colors observed experimentally are due to the different optical paths produced by the different orientations of the molecules. Moreover, from the Ginzburg-Landau equation, one deduces that the interaction between vortices is symmetric [7,71], but it has been reported that the speeds of \pm 1 umbilic defects in the process of collision are different [70]. Numerical simulations that consider the dynamic of the nematic liquid crystal show the same result, where the speed asymmetry arises from backflow effects and anisotropy in the elastic constants [70].

For the purpose of the preceding discussion we assume that $\mu=1$. Let us now consider the effect of the anisotropy of the elastic constants $(\delta \neq 0)$. From the point of view of symmetries, Eq. (5) and the free energy $\mathcal{E}$ are still invariant under spatial translation, but phase invariance and coordinates rotation are no longer valid symmetries. They are replaced by a joint symmetry $A(\zeta, t) \rightarrow A\left(\zeta e^{-i \varphi_{0}}, t\right) e^{i \varphi_{0}}$, where $\zeta$ is the complex variable that represents the Cartesian plane, i.e., $\zeta=x+i y$.

Figure 29 illustrates vortices with positive and negative topological charge found in the anisotropic Ginzburg-Landau equation [Eq. (A3)]. Note that from the nullcline field $\psi(r, t)$, it is not possible to differentiate these vortices, compared to the magnitude field $|A(r, t)|$, where they are distinguishable (see Fig. 29). For the vortex with charge +1 , the modulus remains rotationally invariant, while for the -1 vortex, the rotational invariance around the core is broken by the fourfold symmetry. Indeed, in a single color map representation of $|A|$, one can identify the positive and negative charges on their circular and cross structures, respectively [see Fig. 29(f)]. Note that when one increases the anisotropy, the size of the cross structure grows.

By introducing the ansatz $A\left(r, \theta,\left\{\varphi_{0}\right\}\right)=R(r) e^{i\left(\theta+\varphi_{0}\right)}$ in the anisotropic Ginzburg-Landau equation [Eq. (A3)], for the vortex solution with positive topological charge, one obtains the following set of scalar equations [64]:

$$
0=\mu_{0} R-R^{3}+\left(1+\delta e^{-2 i \varphi_{0}}\right)\left(\frac{d^{2} R}{d^{2} r}+\frac{1}{r} \frac{d R}{d r}-\frac{R}{r^{2}}\right),
$$



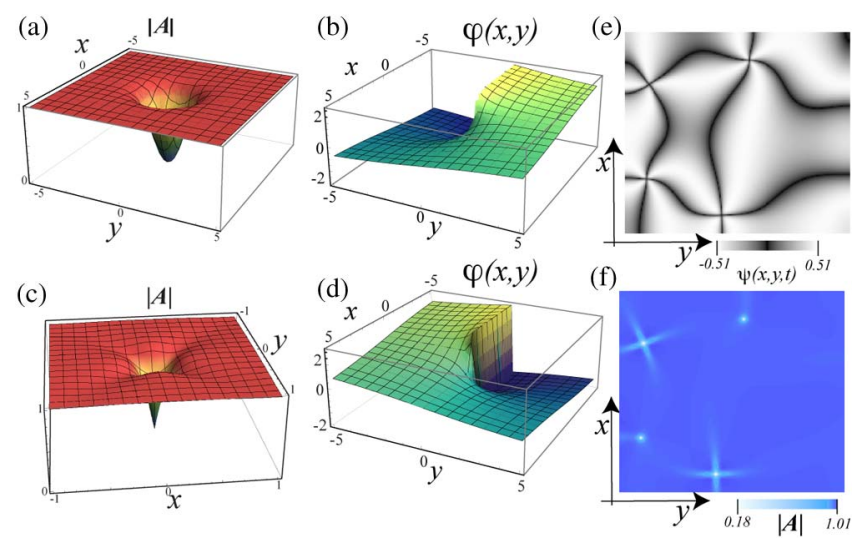

Vortex solution of the anisotropic Ginzburg-Landau equation [Eq. (A3)] with $\mu=1$ and $\delta=0.7$ (from numerical simulations). Structure of the (a) magnitude and (b) phase of the positive vortex. Structure of the (c) magnitude and (d) phase of the negative vortex. (e) Color map of nullcline field $\psi(x, y, t)=\operatorname{Re}(A) \operatorname{Im}(A)$, and (f) modulus of the amplitude $A$ at given time. [Adapted from Fig. 3 in [64], copyright 2014, with permission from the American Physical Society.]

$$
0=\delta \sin 2 \varphi_{0}\left(\frac{d^{2} R}{d^{2} r}+\frac{1}{r} \frac{d R}{d r}-\frac{R}{r^{2}}\right)
$$

From Eq. (A9), the only possible way to obtain a nontrivial solution is to consider the phase parameter satisfying $\sin 2 \varphi_{0}=0$, which gives the discrete solutions $\varphi_{0}=\{0, \pi / 2, \pi, 3 \pi / 2\}$. Therefore, from the continuous family of possible phase jumps, only four possibilities survive. On the other hand, the equation for the magnitude of the amplitude reads

$$
0=\mu_{0} R-R^{3}+\left(1+\delta \cos 2 \varphi_{0}\right)\left(\frac{d^{2} R}{d^{2} r}+\frac{1}{r} \frac{d R}{d r}-\frac{R}{r^{2}}\right) .
$$

Since $\varphi_{0}=\{0, \pi / 2, \pi, 3 \pi / 2\}$, we must have $\cos 2 \varphi_{0}= \pm 1$. Rescaling the space by the factor $\sqrt{1 \pm \delta}$, the above equation becomes Eq. (A7). Therefore, the isotropic positive vortex has the form

$$
A=R_{v}^{ \pm}\left(\frac{r}{\sqrt{1 \pm \delta}}\right) e^{i\left(\theta+\frac{\pi}{4} \mp \frac{\pi}{4}+n \pi\right)},
$$

with $R_{v}$ the magnitude of the vortex solution of the Ginzburg-Landau equation [Eq. (5)] and $n=0, \pm 1, \pm 2, \ldots$. Consequently, the anisotropic vortex solution with positive charge corresponds to a simple scaling of the isotropic vortex solution, although, with a finite number of possible phase jumps $\left(\varphi_{0}=\right.$ $\{0, \pi / 2, \pi, 3 \pi / 2\})$, in opposition to the isotropic system, which has an infinite number of solutions parameterized by the continuous parameter $\varphi_{0}$.

Figure 30 illustrates the magnitude of a vortex with positive topological charge for the anisotropic Ginzburg-Landau equation [Eq. (A3)], for positive and negative anisotropy. Note that the difference between the vortices $R_{v}^{+}$and $R_{v}^{-}$in the amplitude is the different sizes of the vortex core. For positive (negative) 

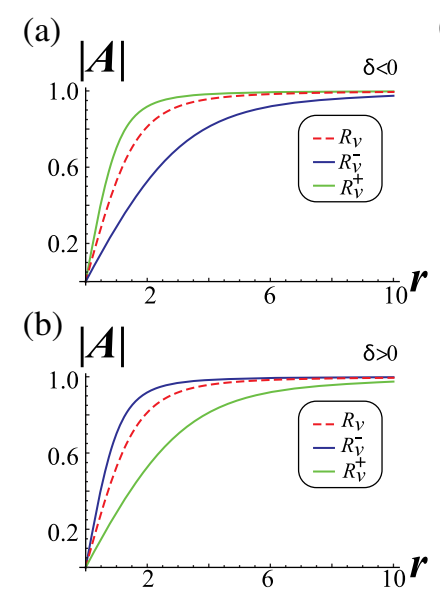

(c)

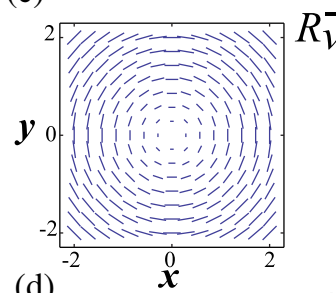

(d)
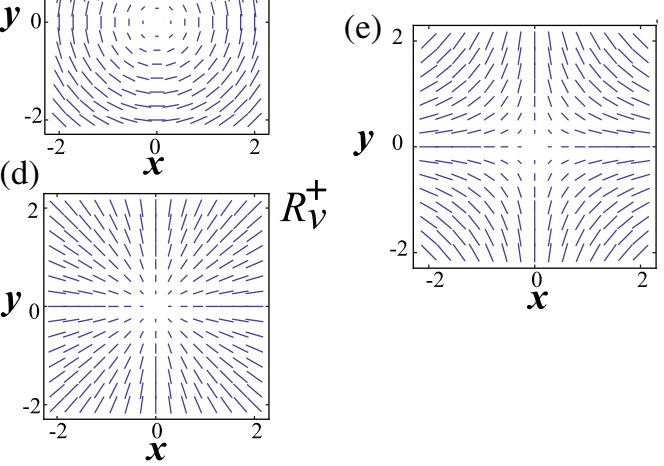

Vortex solution with positive topological charge of the anisotropic GinzburgLandau equation [Eq. (A3)] with $\mu_{0}=1$ and $\delta=0.1$. Magnitude of the vortex with (a) positive and (b) negative anisotropy. The dashed curve stands for the magnitude of the vortex for isotropic systems $(\delta=0)$. (c) and (d) Schematic representations of the orientation field $A\left(r, \theta,\left\{\varphi_{0}\right\}\right)$ for different values of $\varphi_{0}: R_{v}^{-}\left(\varphi_{0}=\pi / 2\right)$ and $R_{v}^{+}\left(\varphi_{0}=0\right)$. (e) Schematic representation of the orientation amplitude field for the negative topological charge. [Adapted from Fig. 4 in [64], copyright 2014, with permission from the American Physical Society.]

anisotropy, the largest core is for vortex $R_{v}^{+}\left(R_{v}^{-}\right)$. Also, because of the different $\varphi_{0}$, both vortices represent different configurations for the director orientation [see Figs. 30(c) and 30(d)].

It is worth noting that it is known, from the variational approach to the Frank free energy, that the elastic anisotropy allows a discrete number of four possible phase jumps for umbilics with positive topological charge [60,81]. These features are recovered by the analytical expression in Eq. (A11) [64]. In the context of self-organization of an array of microtubules interacting via molecular motors, similar configurations have been numerically found for the orientational field with $\varphi_{0}=0$ and $\varphi_{0}=\pi / 2$, which have been named, respectively, the aster and the ideal vortex [74]. Notice that these configurations and their continuous deformation are vortex solutions, like Frank remarked at the dawn of the theory of liquid crystals [63].

To study the existence, stability properties, and bifurcation diagram of the vortex solution with positive topological charge, one can analyze the properties of the free energy $\mathcal{E}$ [Eq. (A5). Using the vortex solution $A=R_{v}^{ \pm}(r / \sqrt{1 \pm \delta}) e^{i\left(\theta+\varphi_{0}\right)}$, where the \pm sign represents + for $\varphi_{0}=\{0, \pi\}$ and - for $\varphi_{0}=\{\pi / 2,3 \pi / 2\}$, and taking a finite large domain $\Omega$, one obtains, after straightforward calculations and following the same strategy presented in Ref. [7], the energy of the vortex with positive topological charge [64]:

$$
\mathcal{E}=\pi \ln \left(\frac{L}{a_{0} \sqrt{1 \pm \delta}}\right)+\frac{\pi(1 \pm \delta)}{2} \pm \pi \delta\left(\ln \left(\frac{L}{a_{0} \sqrt{1 \pm \delta}}\right)+1\right) .
$$

Figure 31 shows the energy for the two different vortices with positive topological charge (two respective signs). The lines and geometrical symbols represent, 
respectively, the energy obtained using Eq. (A12) and obtained from numerical simulations of Eq. (A3). The numerical results show quite good agreement with the analytical expressions. Note that this figure shows that the scaling $\sqrt{1 \pm \delta}$ that makes the core smaller is the one with less energy and is, therefore, preferred by the system. Therefore, if $\delta<0(\delta>0)$ the solution with minimal energy is the one with $\varphi_{0}=\{0, \pi\} \quad\left(\varphi_{0}=\{\pi / 2,3 \pi / 2\}\right)$. Numerical simulations of the anisotropic Ginzburg-Landau equation [Eq. (A3)] show that the vortices with positive topological charge and large core are unstable. Thus, the stable vortices are those with small core. The respective stability of these solutions is represented by continuous (stable) and dashed (unstable) lines in Fig. (31). One expects that vortices with small core are the more stable, because the energy privileges the uniform state $|A|^{2}=\mu$.

The above analysis shows that there are two positive vortex solutions that exist for every value of $\delta$. These phase singularity solutions exchange stability in the isotropic limit $(\delta=0)$, where $\varphi_{0}=\{0, \pi\}$ goes from a stable to an unstable solution, and vice versa for $\varphi_{0}=\{\pi / 2,3 \pi / 2\}$. The mechanism through which these solutions exchange stability is not by the usual collision of solutions of the transcritical bifurcation [83,84], but rather by passing through a degenerate point at $\delta=0$, where an infinite number of solutions exists and $\varphi_{0}$ can take any continuous value between 0 and $2 \pi$. Hence, this bifurcation is a degenerate

\section{Figure 31}

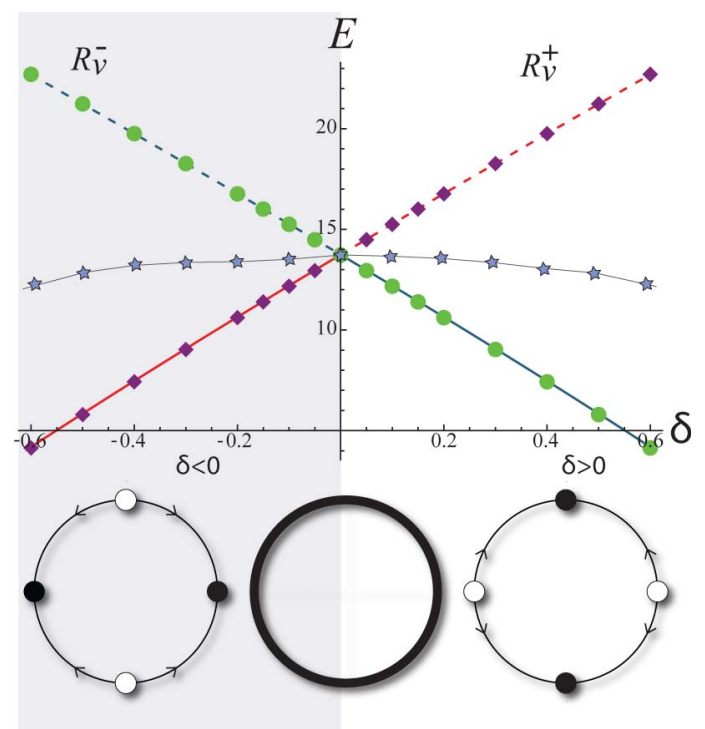

Energy of the positive vortex solutions for different jump phase $\varphi_{0}$ as function of $\delta$. Numerical results obtained from vortex solutions of Eq. (16) are shown by the geometrical symbols (circles and diamonds) and the theoretical result obtained from expression (25) by continuous and dashed lines. The continuous and dashed line indicate, respectively, the stable and unstable vortex solution with positive topological charge. The star symbols account for the energy of vortex with negative charge. The bottom panel schematically illustrates the bifurcation diagram for the phase jump $\varphi_{0}$, which corresponds to a degenerate transcritical bifurcation. The dark and white circles account for stable and unstable solutions. Reprinted with permission from Clerc et al., Phys. Rev. E 90, 012507 (2014) [64]. Copyright 2014 the American Physical Society. 
transcritical bifurcation and it is schematically shown in the bottom panel of Fig. 31, where the solid and open circles account for stable and unstable solutions, respectively.

The above analysis yields a complete description of vortices with topological charge +1 . As we have mentioned, in vortices with negative topological charge, their rotational invariance around the core is broken by a fourfold symmetry (see Fig. 29). To grasp these phase singularities, one can consider the strategy of perturbative analysis of these solutions for small anisotropy $(\delta \ll 1)$. Hence, one can consider the following ansatz:

$$
A(r, \theta) \approx\left[R_{v}(r)+\delta g(r, \theta)+O\left(\delta^{2}\right)\right] e^{-i[\theta-\delta \Theta(r, \theta)]},
$$

where $g(r, \theta)$ and $\Theta(r, \theta)$ are leading correction functions to the isotropic negative vortex, and with the condition that $\Theta$ has no topological charge, i.e., $\oint_{\Gamma} \nabla \Theta \cdot \mathrm{d} \vec{l}=0$, where the path $\Gamma$ encircles the core of the vortex. Using the above ansatz [Eq. (A13)] in the anisotropic Ginzburg-Landau equation [Eq. (A3)] and taking the leading order in $\delta$, we obtain

$$
\begin{aligned}
0= & e^{-i \theta}\left[\mu g-3 R_{v}^{2} g+\frac{\partial^{2} g}{\partial r^{2}}+2 i \frac{\partial \Theta}{\partial r} \frac{\partial R_{v}}{\partial r}+i R_{v} \frac{\partial^{2} \Theta}{\partial r^{2}}+\frac{1}{r} \frac{\partial g}{\partial r}+\frac{i R_{v}}{r} \frac{\partial \Theta}{\partial r}+\frac{1}{r^{2}} \frac{\partial^{2} g}{\partial \theta^{2}}\right. \\
& \left.-\frac{2 i}{r^{2}} \frac{\partial g}{\partial \theta}+\frac{i R_{v}}{r^{2}} \frac{\partial^{2} \Theta}{\partial \theta^{2}}+\frac{2 R_{v}}{r^{2}} \frac{\partial \Theta}{\partial \theta}-\frac{g}{r^{2}}\right]+e^{3 i \theta}\left[\frac{\partial^{2} R_{v}}{\partial r^{2}}+\frac{3 R_{v}}{r^{2}}-\frac{3}{r} \frac{\partial R_{v}}{\partial r}\right], \quad \text { (A14) }
\end{aligned}
$$

separating, respectively, the real and imaginary parts:

$$
\begin{aligned}
0= & \mu g-3 R_{v}^{2} g+\frac{\partial^{2} g}{\partial r^{2}}+\frac{1}{r} \frac{\partial g}{\partial r}+\frac{1}{r^{2}} \frac{\partial^{2} g}{\partial \theta^{2}}+\frac{2 R_{v}}{r^{2}} \frac{\partial \Theta}{\partial \theta}-\frac{g}{r^{2}} \\
& +\cos (4 \theta)\left[\frac{\partial^{2} R_{v}}{\partial r^{2}}+\frac{3 R_{v}}{r^{2}}-\frac{3}{r} \frac{\partial R_{v}}{\partial r}\right], \\
0= & 2 \frac{\partial \Theta}{\partial r} \frac{\partial R_{v}}{\partial r}+R_{v} \frac{\partial^{2} \Theta}{\partial r^{2}}+\frac{R_{v}}{r} \frac{\partial \Theta}{\partial r}+\frac{R_{v}}{r^{2}} \frac{\partial^{2} \Theta}{\partial \theta^{2}}-\frac{2}{r^{2}} \frac{\partial g}{\partial \theta} \\
& +\sin (4 \theta)\left[\frac{\partial^{2} R_{v}}{\partial r^{2}}+\frac{3 R_{v}}{r^{2}}-\frac{3}{r} \frac{\partial R_{v}}{\partial r}\right] .
\end{aligned}
$$

The $\theta$-dependence is easily addressed by doing variable separation, by setting $g(r, \theta)=g_{4}(r) \cos (4 \theta)$ and $\Theta(r, \theta)=\theta_{4}(r) \sin (4 \theta)$. Thus, one obtains the following set of equations for the radial dependency [64]:

$0=\mu g_{4}-3 R_{v}^{2} g_{4}+\frac{\partial^{2} g_{4}}{\partial r^{2}}+\frac{1}{r} \frac{\partial g_{4}}{\partial r}-\frac{16 g_{4}}{r^{2}}+\frac{8 R_{v} \theta_{4}}{r^{2}}-\frac{g_{4}}{r^{2}}+\frac{\partial^{2} R_{v}}{\partial r^{2}}+\frac{3 R_{v}}{r^{2}}-\frac{3}{r} \frac{\partial R_{v}}{\partial r}$, $0=2 \frac{\partial \theta_{4}}{\partial r} \frac{\partial R_{v}}{\partial r}+R_{v} \frac{\partial^{2} \theta_{4}}{\partial r^{2}}+\frac{R_{v}}{r} \frac{\partial \theta_{4}}{\partial r}+\frac{8 g_{4}}{r^{2}}-\frac{16 R_{v} \theta_{4}}{r^{2}}+\frac{\partial^{2} R_{v}}{\partial r^{2}}+\frac{3 R_{v}}{r^{2}}-\frac{3}{r} \frac{\partial R_{v}}{\partial r}$.

As $r \rightarrow \infty$, the solution of this set of equations behaves as follows: $g_{4}(r) \rightarrow 9 / 4 r^{2}, \theta_{4}(r) \rightarrow 3 / 16$. Then, the phase correction converges to a constant value. Using a variational approach to the Frank free energy far from the core of the vortex, neglecting the spatial dependence, and considering a modal 
angular expansion, one can recover the values of $\theta_{4}=3 / 16$ and $g_{4}=0$ [81]. However, this ansatz does not allow us to characterize the spatial structure of the negative vortex solution. Asymptotically, the correction of the magnitude of the amplitude, $g_{4}(r)$, decreases as the inverse of the square of the distance. A numerical solution for $g_{4}(r)$ has quite good agreement with the above asymptotic expression [64].

Note that in the perturbative analysis the phase jump, $\varphi_{0}$, is not predetermined, because if we consider a more general ansatz,

$$
A\left(r, \theta, \varphi_{0}\right) \approx\left[R_{v}(r)+\delta g(r, \theta)\right] e^{-i\left[\theta+\varphi_{0}-\delta \Theta(r, \theta)\right]},
$$

the previous analysis remains the same by setting $g(r, \theta)=g_{4}(r) \cos \left(4 \theta-2 \varphi_{0}\right)$ and $\Theta(r, \theta)=\theta_{4}(r) \sin \left(4 \theta-2 \varphi_{0}\right)$. Therefore, the vortex solution with negative topological charge is a continuous family of solutions parametrized by $\varphi_{0}$. Furthermore, when the anisotropy parameter $\delta$ is modified numerically, the vortex does not exhibit any bifurcation.

By using the vortex solution with negative topological charge obtained numerically from the anisotropic Ginzburg-Landau equation [Eq. (A3)] and evaluating the free energy $\mathcal{E}$ [Eq. (A5)], we can reveal the dependence of the free energy as a function of the anisotropy, $\mathcal{E}(\delta)$. Figure 31 shows this function for various critical points of $\mathcal{E}$; the numerical evaluation of this energy is represented by stars. The energy of the vortex with negative charge is exactly like the positive one, except at $\delta=0$. The vortex with positive topological charge is always more stable for anisotropic nematic liquid crystals. It is worth noting that vortices are always created by pairs to conserve the topological charge, even though one vortex has more energy than the other. Furthermore, the scenario of the collision of opposite vortices described by the isotropic Ginzburg-Landau (see $[7,71]$ and references therein) does not account for the whole picture of the collision of opposite nematic umbilics, as is shown in [70]. The characterization of vortex interaction in the anisotropic Ginzburg-Landau equation is an open problem.

Therefore, the anisotropic elasticity is responsible for the dissimilarity of the defects with different topological charges. One possible experimental protocol for characterizing several properties of these nematic umbilics is through the use of crossed circular polarizers [82] and modification of the elastic constants by changing the temperature. Temperature allows us to handle the values of the elastic anisotropy constants. In particular, the elastic constants are quite sensitive to temperature near the nematic-smectic transition [54].

\section{Acknowledgment}

We acknowledge financial support from the ANR international program, project ANR-2010-INTB-402-02 (ANR-CONICYT39), "COLORS." MGC is thankful for the financial support from FONDECYT projects 1120320 and 1150507. RB is grateful for financial support from FONDECYT projects 1120320, 1150507, and 3140577. EVH acknowledges a Master fellowship from CONICYT contract 221320023 and financial support from the DPP of the University of Chile. 


\section{References}

1. J. F. Nye, R. G. Kyte, and D. C. Threlfall, "Proposal for measuring the movement of a large ice sheet by observing radio echoes," J. Glaciol. 11, 319-325 (1972).

2. M. E. R. Walford, "Glacier movement measured with a radio echo technique," Nature 239, 93-95 (1972).

3. J. F. Nye, M. V. Berry, and M. E. R. Walford, "Measuring the change in thickness of the Antarctic ice sheet," Nature 240, 7-9 (1972).

4. J. Nye and M. Berry, "Dislocations in wave trains," Proc. R. Soc. Lond. A. 336, 165-190 (1974).

5. P. Hariharan, Basics of Interferometry (Elsevier/Academic, 2007).

6. C. Kittel, Introduction to Solid State Physics (Wiley, 1996).

7. L. Pismen, Vortices in Nonlinear Fields (Oxford University, 1999).

8. M. S. Soskin and M. V. Vasnetov, "Singular optics," in Progress in Optics, E. Wolf, ed. (Elsevier, 2001), Vol. 42, pp. 219-276.

9. K. Staliunas and V. J. Sanchez-Morcillo, Transverse Patterns in Nonlinear Optical Resonators (Springer-Verlag, 2003).

10. M. R. Dennis, K. O’Holleran, and M. J. Padgett, "Singular optics: optical vortices and polarization singularities," in Progress in Optics, E. Wolf, ed. (Elsevier, 2009), Vol. 53, pp. 293-363.

11. R. A. Beth, "Mechanical detection and measurement of the angular momentum of light," Phys. Rev. 50, 115-125 (1936).

12. H. He, M. E. J. Friese, N. R. Heckenberg, and H. Rubinsztein-Dunlop, "Direct observation of transfer of angular momentum to absorptive particles from a laser beam with a phase singularity," Phys. Rev. Lett. 75, 826-829 (1995).

13. A. T. O'Neil, I. MacVicar, L. Allen, and M. J. Padgett, "Intrinsic and extrinsic nature of the orbital angular momentum of a light beam," Phys. Rev. Lett. 88, 053601 (2002).

14. A. Y. Bekshaev, M. S. Soskin, and M. V. Vasnetsov, Paraxial Light Beams with Angular Momentum (Nova Science, 2008).

15. P. Coullet, L. Gil, and F. Rocca, "Optical vortices," Opt. Commun. 73, 403-408 (1989).

16. F. T. Arecchi, G. Giacomelli, P. L. Ramazza, and S. Residori, "Vortices and defect statistics in two-dimensional optical chaos," Phys. Rev. Lett. 67, 3749-3752 (1991).

17. M. Brambilla, F. Battipede, L. A. Lugiato, V. Penna, F. Prati, C. Tamm, and C. O. Weiss, "Transverse laser patterns. I. Phase singularity crystals," Phys. Rev. A 43, 5090-5113 (1991).

18. C. O. Weiss, H. R. Telle, K. Staliunas, and M. Brambilla, "Restless optical vortex," Phys. Rev. A 47, R1616-R1619 (1993).

19. A. M. Yao and M. J. Padgett, "Orbital angular momentum: origins, behavior and applications," Adv. Opt. Photon. 3, 161-204 (2011).

20. L. Allen, M. W. Beijersbergen, R. J. C. Spreeuw, and J. P. Woerdman, "Orbital angular momentum of light and the transformation of LaguerreGaussian laser modes," Phys. Rev. A 45, 8185-8189 (1992).

21. D. G. Grier, "A revolution in optical manipulation," Nature 424, 810-816 (2003).

22. V. G. Shvedov, A. V. Rode, Y. V. Izdebskaya, A. S. Desyatnikov, W. Krolikowski, and Y. S. Kivshar, "Giant optical manipulation," Phys. Rev. Lett. 105, 118103 (2010). 
23. M. Padgett and R. Bowman, "Tweezers with a twist," Nat. Photonics 5, 343-348 (2011).

24. J. Hamazaki, R. Morita, K. Chujo, Y. Kobayashi, S. Tanda, and T. Omatsu, "Optical-vortex laser ablation," Opt. Express 18, 2144-2151 (2010).

25. O. J. Allegre, W. Perrie, S. P. Edwardson, G. Dearden, and K. G. Watkins, "Laser microprocessing of steel with radially and azimuthally polarized femtosecond vortex pulses," J. Opt. 14, 085601 (2012).

26. C. Hnatovsky, V. G. Shvedov, W. Krolikowski, and A. V. Rode, "Materials processing with a tightly focused femtosecond laser vortex pulse," Opt. Lett. 35, 3417-3419 (2010).

27. H. H. Arnaut and G. A. Barbosa, "Orbital and intrinsic angular momentum of single photons and entangled pairs of photons generated by parametric down-conversion," Phys. Rev. Lett. 85, 286-289 (2000).

28. J. Wang, J. Y. Yang, I. M. Fazal, N. Ahmed, Y. Yan, H. Huang, Y. Ren, Y. Yue, S. Dolinar, M. Tur, and A. E. Willner, "Terabit free-space data transmission employing orbital angular momentum multiplexing," Nat. Photonics 6, 488-496 (2012).

29. G. Gibson, J. Courtial, M. J. Padgett, M. Vasnetsov, V. Pas'ko, S. M. Barnett, and S. Franke-Arnold, "Free-space information transfer using light beams carrying orbital angular momentum," Opt. Express 12, 5448-5456 (2004).

30. G. K. L. Wong, M. S. Kang, H. W. Lee, F. Biancalana, C. Conti, T. Weiss, and P. St. J. Russell, "Excitation of orbital angular momentum resonances in helically twisted photonic crystal fiber," Science 337, 446-449 (2004).

31. S. Ramachandran and P. Kristensen, "Optical vortices in fiber," Nano Photon. 2, 455-474 (2013).

32. F. Tamburini, G. Anzolin, G. Umbriaco, A. Bianchini, and C. Barbieri, "Overcoming the Rayleigh criterion limit with optical vortices," Phys. Rev. Lett. 97, 163903 (2006).

33. S. R. Nersisyan, N. V. Tabiryan, D. Mawet, and E. Serabyn, "Improving vector vortex waveplates for high- contrast coronagraphy," Opt. Express 21, 8205-8213 (2013).

34. M. W. Beijersbergen, L. Allen, H. E. L. O. van der Veen, and J. P. Woerdman, "Astigmatic laser mode converters and transfer of orbital angular momentum," Opt. Commun. 96, 123-132 (1993).

35. Z. Sacks, D. Rozas, and G. A. Swartzlander, "Holographic formation of optical-vortex filaments," J. Opt. Soc. Am. B 15, 2226-2234 (1998).

36. J. Arlt, K. Dholakia, L. Allen, and M. J. Padgett, "The production of multiringed Laguerre-Gaussian modes by computer-generated holograms," J. Mod. Opt. 45, 1231-1237 (1998).

37. R. K. Tyson, M. Scipioni, and J. Viegas, "Generation of an optical vortex with a segmented deformable mirror," Appl. Opt. 47, 6300-6306 (2008).

38. M. Beresna, M. Gecevicius, P. G. Kazansky, and T. Gertus, "Radially polarized optical vortex converter created by femtosecond laser nanostructuring of glass," Appl. Phys. Lett. 98, 201101 (2011).

39. D. Voloschenko and O. D. Lavrentovich, "Optical vortices generated by dislocations in a cholesteric liquid crystal," Opt. Lett. 25, 317319 (2000).

40. L. Marrucci, C. Manzo, and D. Paparo, "Optical spin-to-orbital angular momentum conversion in inhomogeneous anisotropic media," Phys. Rev. Lett. 96, 163905 (2006).

41. E. Brasselet, N. Murazawa, H. Misawa, and S. Juodkazis, "Optical vortices from liquid crystal droplets," Phys. Rev. Lett. 103, 103903 (2009). 
42. E. Brasselet and C. Loussert, "Electrically controlled topological defects in liquid crystals as tunable spin-orbit encoders for photons," Opt. Lett. 36, 719 (2011).

43. E. Brasselet, "Tunable optical vortex arrays from a single nematic topological defect," Phys. Rev. Lett. 108, 087801 (2012).

44. A. Y. Bekshaev and S. V. Sviridova, "Effects of misalignments in the optical vortex transformation performed by holograms with embedded phase singularity," Opt. Commun. 283, 4866-4876 (2010).

45. R. Barboza, U. Bortolozzo, G. Assanto, E. Vidal-Henriquez, M. G. Clerc, and S. Residori, "Vortex induction via anisotropy stabilized light-matter interaction," Phys. Rev. Lett. 109, 143901 (2012).

46. R. Barboza, U. Bortolozzo, G. Assanto, E. Vidal-Henriquez, M. G. Clerc, and S. Residori, "Harnessing optical vortex lattices in nematic liquid crystals," Phys. Rev. Lett. 111, 93902 (2013).

47. I. I. Smalyukh, Y. Lansac, N. A. Clark, and R. P. Trivedi, "Threedimensional structure and multistable optical switching of triple-twisted particle-like excitations in anisotropic fluids," Nat. Mater. 9, 139-145 (2010).

48. B. Yang and E. Brasselet, "Arbitrary vortex arrays realized from optical winding of frustrated chiral liquid crystals," J. Opt. 15, 044021 (2013).

49. Y. H. Kim, D. K. Yoon, M. C. Choi, H. S. Jeong, M. W. Kim, O. D. Lavrentovich, and H.-T. Jung, "Confined self-assembly of toric focal conic domains (the effects of confined geometry on the feature size of toric focal conic domains)," Langmuir 25, 1685-1691 (2009).

50. Y. H. Kim, D. K. Yoon, H. S. Jeong, O. D. Lavrentovich, and H.-T. Jung, "Smectic liquid crystal defects for self-assembling of building blocks and their lithographic applications," Adv. Funct. Mater. 21, 610-627 (2011).

51. B. Son, S. Kim, Y. Ho Kim, K. Käläntär, H.-M. Kim, H.-S. Jeong, S. Q. Choi, J. Shin, H.-T. Jung, and Y.-H. Lee, "Optical vortex arrays from smectic liquid crystals," Opt. Express 22, 4699-4704 (2014).

52. C. Loussert, K. Kushnir, and E. Brasselet, "Q-plates micro-arrays for parallel processing of the photon orbital angular momentum," Appl. Phys. Lett. 105, 121108 (2014).

53. P. Pieranski, B. Yang, L.-J. Burtz, A. Camu, and F. Simonetti, "Generation of umbilics by magnets and flows," Liq. Cryst. 40, 1593-1608 (2013).

54. S. Chandrasekhar, Liquid Crystals (Cambridge University, 1977).

55. P. G. de Gennes and J. Prost, The Physics of Liquid Crystals, 2nd ed. (Oxford, 1993).

56. P. Oswald and P. Pieranski, Nematic and Cholesteric Liquid Crystals (Taylor \& Francis, 2005).

57. D.-K. Yang and S.-T. Wu, Fundamentals of Liquid Crystals Devices (Wiley, 2006).

58. I. C. Khoo, Liquid Crystals: Physical Properties and Nonlinear Optical Phenomena, 2nd ed. (Wiley, 2006).

59. L. Marrucci, "Generation of helical modes of light by spin-to-orbital angular momentum conversion in inhomogeneous liquid crystals," Mol. Cryst. Liq. Cryst. 488, 148-162 (2008).

60. A. Rapini, "Umbilics: static properties and shear-induced displacements," J. Phys. France 34, 629-633 (1973).

61. O. Lehmann, "Über fliessende krystalle," Z. Phys. Chem. 4, 462-472 (1889). 
62. G. Friedel, "The mesomorphic states of matter," Ann. Phys. 18, 273-474 (1922).

63. F. C. Frank, "Liquid crystals. On the theory of liquid crystals," Discuss. Faraday Soc. 25, 19-28 (1958).

64. M. G. Clerc, E. Vidal-Henriquez, J. D. Davila, and M. Kowalczyk, "Symmetry breaking of nematic umbilical defects through an amplitude equation," Phys. Rev. E 90, 012507 (2014).

65. T. Frisch, S. Rica, P. Coullet, and J. M. Gilli, "Spiral waves in liquid crystal," Phys. Rev. Lett. 72, 1471-1474 (1994).

66. T. Frisch, "Spiral waves in nematic and cholesteric liquid crystals," Phys. D 84, 601-614 (1995).

67. G. Nicolis and I. Prigogine, Self-Organization in Non Equilibrium Systems (Wiley, 1977).

68. L. M. Pismen, Patterns and Interfaces in Dissipative Dynamics, Springer Series in Synergetics (Springer, 2006).

69. M. C. Cross and P. C. Hohenberg, "Pattern formation outside of equilibrium," Rev. Mod. Phys. 65, 851-1112 (1993).

70. I. Dierking, O. Marshall, J. Wright, and N. Bulleid, "Annihilation dynamics of umbilical defects in nematic liquid crystals under applied electric fields," Phys. Rev. E 71, 061709 (2005).

71. R. Barboza, T. Sauma, U. Bortolozzo, G. Assanto, M. G. Clerc, and S. Residori, "Characterization of the vortex-pair interaction law and nonlinear mobility effects," New J. Phys. 15, 013028 (2013).

72. R. Barboza, U. Bortolozzo, M. G. Clerc, S. Residori, and E. VidalHenriquez, "Light-matter interaction induces a single positive vortex with swirling arms," Phil. Trans. R. Soc. A 372, 20140019 (2014).

73. M. S. Soskin, V. G. Denisenko, and R. I. Egorov, "Singular Stokes-polarimetry as new technique for metrology and inspection of polarized speckle fields," Proc. SPIE 5458, 79-85 (2004).

74. I. S. Aranson and L. S. Tsimring, "Theory of self-assembly of microtubules and motors," Phys. Rev. E 74, 031915 (2006).

75. V. L. Ginzburg and L. D. Landau, "On the theory of superconductivy," Sov. Phys. J. Exp. Theor. Phys. 20, 1064 (1950).

76. I. Aranson and L. Kramer, "The world of the complex Ginzburg-Landau equation," Rev. Mod. Phys. 74, 99-143 (2002).

77. V. L. Ginzburg and L. P. Pitaeskii, "On the theory of superfluidity," Sov. Phys. J. Exp. Theor. Phys. 34, 858 (1959).

78. L. P. Pitaevskii, "Vortex lines in an imperfect Bose gas," Sov. Phys. J. Exp. Theor. Phys. 13, 451-454 (1961).

79. E. P. Gross, "Structure of a quantized vortex in boson systems," Nuovo Cimento 20, 454477 (1960).

80. F. Bethuel, H. Brezis, and F. Helein, Ginzburg-Landau Vortices (Springer, 1994).

81. A. Saupe, "Disclinations and properties of the directorfield in nematic and cholesteric liquid crystals," Mol. Cryst. Liq. Cryst. 21, 211-238 (1973).

82. R. B. Meyer, "On the existence of even indexed disclinations in nematic liquid crystals," Philos. Mag. 27(2), 405-424 (1973).

83. S. H. Strogatz, Nonlinear Dynamics and Chaos: with Applications to Physics, Biology, Chemistry and Engineering (Addison-Wesley, 1994).

84. J. D. Crawford, "Introduction to bifurcation theory," Rev. Mod. Phys. 63, 991-1037 (1991). 


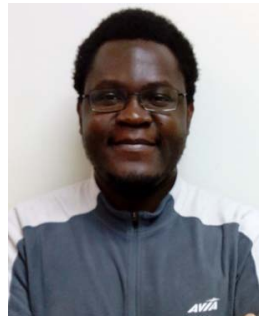

R. Barboza is currently a Postdoctoral Researcher at the University of Chile, Chile. He received his Laurea in Electronic Engineering in 2005 at the University "La Sapienza" in Rome, Italy and in 2009 a Laurea Specialistica in Electronic Engineering at Politecnico di Milano. He obtained in 2013 a Ph.D. degree in Electronics at the University of Roma Tre (Italy) and a Ph.D. in Physics at the Universit de Nice Sophia-Antipolis. His research interests include nonlinear optics, soft condensed matter, liquid crystals, singular optics, and liquid-crystal device engineering.

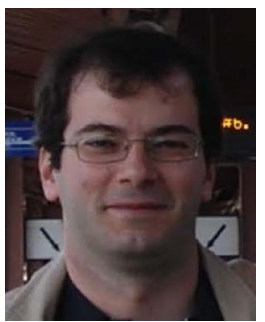

U. Bortolozzo is currently an Associate Professor at the University of Nice-Sophia Antipolis, France. He obtained a master's degree in Telecommunication Engineering at the University of Padova in 1999. In 2001 he was enrolled as a researcher at the National Institute of Applied Optics (INOA) in Florence, and in 2005 he got a Ph.D. in Complex Systems at the University of Florence (Italy) and in Physics at the University of Nice (France). After his thesis he has been a postdoctoral researcher at the Ecole Normale Supérieure in Paris. In 2009, he joined the University of Nice-Sophia Antipolis. His main research interests include liquid crystals, soft matter, dynamical holography, and adaptive optics.

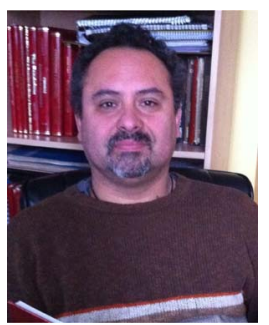

M. G. Clerc is a full professor of Physics at the Universidad de Chile, Chile. He received his undergraduate and master's degrees in Physics at the Universidad de Chile, in 1994 and 1996, and his Ph.D. in Physics from the University of Nice and Sophia-Antipolis, France, in 1999, followed by his postdoctoral appointment at the California Institute of Technology, CALTECH, USA. His research interests are focused on understanding the robust phenomena of nonequilibrium systems, which include soft matter, liquid crystals, nonlinear optic, mechanics, fluid dynamics, magnetics, granular media, population dynamics, statistical mechanics, stochastic processes, bifurcation, and dynamical systems theory.

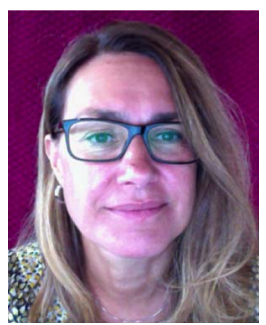

S. Residori is currently a Research Director of the Centre National de la Recherche Scientifique (CNRS) at the Institut Non Linaire de Nice (INLN) in Nice, France. She received a Laurea degree in Physics at the University of Bologna, Italy, in 1989, and in 1993 she obtained a Ph.D. in Nonlinear Optics at the University of Florence. In 1994 she was enrolled as a permanent researcher at the National Institute of Optics in Italy, and in 1998 she moved

to France as a Senior Research Scientist at the CNRS. Her research interests include soft matter, liquid crystals, optical wave mixing, optical vortices, and light propagation in disordered media. 


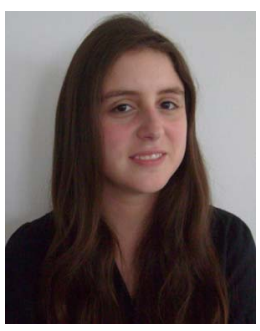

E. Vidal-Henriquez received her undergraduate degrees in Physics and Computer Science from Universidad de Chile in 2012 and her master's degree in Physics in 2014 from Universidad de Chile. In 2015 she received a DAAD scholarship to conduct her Ph.D. studies at the University of Göttingen, which she expects to begin in October 2015. Her main research interests are nonlinear dynamical systems, particularly nonlinear optics, liquid crystals, mechanics, and biocomplexity, among others. 